Pacific

Journal of

Mathematics

FUSION SYMMETRIC SPACES AND SUBFACTORS

HANS WENZL

Volume 259 No. 2

October 2012 


\title{
FUSION SYMMETRIC SPACES AND SUBFACTORS
}

\author{
HANS WENZL
}

\begin{abstract}
We construct analogs of the embedding of orthogonal and symplectic groups into unitary groups in the context of fusion categories. At least some of the resulting module categories also appear in boundary conformal field theory. We determine when these categories are unitarizable, and explicitly calculate the index and principal graph of the resulting subfactors.
\end{abstract}

This paper is a sequel of our previous paper [Wenzl 2012], where we introduced a $q$-deformation of Brauer's centralizer algebra for orthogonal and symplectic groups; this algebra had already appeared more or less before in [Molev 2003]; see also the discussion in [Wenzl 2012]. It is motivated by finding a deformation of orthogonal or symplectic subgroups of a unitary group that is compatible with the standard quantum deformation of the big group. This has been done before on the level of coideal subalgebras of Hopf algebras by Letzter. However, our categorical approach also allows us to extend this to the level of fusion tensor categories, where we find finite analogs of symmetric spaces related to the already mentioned groups. Moreover, we can establish $C^{*}$ structures, necessary for the construction of subfactors, in this categorical setting; this is not so obvious to see in the setting of coideal algebras.

It is well-known how one can use a subgroup $H$ of a (for simplicity here) finite group $G$ to construct a module category of the representation category $\operatorname{Rep} G$ of $G$. This module category also appears in the context of subfactors of $\mathrm{II}_{1}$ von Neumann factors as follows: Let $R$ be the hyperfinite $\mathrm{II}_{1}$ factor, and let $\mathcal{N}=R^{G} \subset \mathcal{M}=R^{H}$ be the fixed points under outer actions of $G$ and $H$. Then the category of $\mathcal{N}-\mathcal{N}$ bimodules is equivalent to $\operatorname{Rep} G$, and the module category is given via the $M-\mathcal{N}$ bimodules of the inclusion $\mathcal{N} \subset \mathcal{M}$; its simple objects are labeled by the irreducible representations of $H$. In particular, an important invariant called the principal graph of the subfactor is determined by the restriction rules for representations from $G$ to $H$. Important examples of subfactors were constructed from fusion categories whose Grothendieck semirings are quotients of the ones of semisimple Lie groups. So a natural question to ask is whether one can perform a similar construction in this context. More precisely, can we find restriction rules for type $A$ fusion categories

MSC2010: 46L10.

Keywords: module tensor categories, subfactors, q-Brauer algebra. 
which describe a subfactor as before, and which will approach in the classical limit the usual restriction rules from $\mathrm{U}(N)$ to $\mathrm{O}(N)$.

We answer this question in the positive in this paper via a fairly elementary construction. We show that certain semisimple quotients of the $q$-Brauer algebras have a $C^{*}$ structure and contain $C^{*}$-quotients of Hecke algebras of type $A$. The subfactor is then obtained as the closure of inductive limits of such algebras. Due to its close connection to Lie groups, we can give very explicit general formulas for its index and its first principal graph. Observe that the Lie algebra $\mathfrak{s l}_{N}$ decomposes as an $\mathfrak{s o}_{N}$ module into the direct sum $\mathfrak{s o}_{N} \oplus \mathfrak{p}$, where $\mathfrak{p}$ is a simple $\mathfrak{s o}_{N}$-module. Then, the index can be expressed explicitly in terms of the weights of $\mathfrak{p}$; see Theorem 3.4. As before in the group case, it can be interpreted as the quotient of the dimension of the given fusion category by the sum of the squares of $q$ dimensions of representations of orthogonal or symplectic subgroups whose labels are in the alcove of a certain affine reflection group; however in our case, there is no corresponding tensor category for the denominator, and the $q$-dimensions differ from the ones of the corresponding quantum groups. Also, the restriction rules for the corresponding bimodules of this subfactor, the first principal graph, can be derived from the classical restriction rules via an action of the already mentioned affine reflection group, similarly as it was done before for tensor product rules for fusion categories. However, in our case, the affine reflection comes from the highest short root of the corresponding Lie algebra in the nonsimply laced case; it is also different from the one for fusion categories in the even-dimensional orthogonal case.

Not surprisingly for such a basic question, many related results have been obtained before in the study of subfactors, tensor categories and boundary conformal field theory. For $N=2$, we obtain the Goodman-de la Harpe-Jones subfactors for Dynkin diagrams $D_{n}$. Subgroups and module categories in connection with SU(3) and SU(4) fusion categories have been studied by Ocneanu [2002] and by Evans and Pugh [2011]; our examples for $N=3$ and $N=4$ appear among the series in these works. The research in this and the just-mentioned papers has also been influenced by closely related results in boundary conformal field theory, which will be discussed in more detail at the end of the paper. Our examples for the odd-dimensional orthogonal group and for symplectic groups also seem to be closely related to type $\mathrm{III}_{1}$ subfactors constructed by Feng Xu [2009] and Antony Wassermann [2010] by completely different methods.

The first chapter mostly contains basic material from subfactor theory which will be needed later. In the second chapter we review and expand material on the $q$-Brauer algebra as defined in [Wenzl 2012]; see also [Molev 2003]. In particular, we define $C^{*}$-structures for certain quotients and use that to construct subfactors. The third chapter is mainly concerned with the finer structure of these subfactors, such as explicit closed formulas for the index and calculation of the first principal 
graph. The same techniques would also extend to other examples, such as the ones in [Xu 2009].

\section{1. $\mathrm{II}_{1}$ factors}

1A. Periodic commuting squares. We will construct subfactors using the setup of periodic commuting squares (going back to work of Jones and Popa) as in [Wenzl 1988a]. More precisely, we assume that we have increasing sequences of finitedimensional $C^{*}$ algebras $A_{1} \subset A_{2} \subset \cdots$ and $B_{1} \subset B_{2} \subset \cdots$ such that $A_{n} \subset B_{n}$ for all $n \in \mathbb{N}$. Let $\Lambda_{n}$ and $\tilde{\Lambda}_{n}$ be labeling sets for the simple components of $B_{n}$ and $A_{n}$, respectively. Let $G_{n}$ be the inclusion matrix for $A_{n} \subset B_{n}$. If we write a minimal idempotent $p_{\mu} \in A_{n, \mu}$ as a sum of minimal mutually commuting idempotents of $B_{n}$, then the entry $g_{\lambda \mu}$ of $G_{n}$ denotes the number of those idempotents which are in $B_{n, \lambda}$. We say that our sequences of algebras are periodic with period $d$ if there exists an $n_{0} \in \mathbb{N}$ such that for any $n>n_{0}$ we have bijections $j$ between $\Lambda_{n}$ and $\Lambda_{n+d}$ as well as between $\tilde{\Lambda}_{n}$ and $\tilde{\Lambda}_{n+d}$ that do not change the inclusion matrices for $A_{n} \subset B_{n}$ as well as for $A_{n} \subset A_{n+1}$ and $B_{n} \subset B_{n+1}$. This means, in particular, that $g_{j(\lambda) j(\mu)}=g_{\lambda \mu}$ for all $\lambda \in \Lambda_{n}, \mu \in \tilde{\Lambda}_{n}, n>n_{0}$.

The trace functional defines inner products on the algebras $A_{n}$ and $B_{n}$ by

$$
\left(b_{1}, b_{2}\right)=\operatorname{tr}\left(b_{1}^{*} b_{2}\right) .
$$

Let $e_{A_{n+1}}$ and $e_{B_{n}}$ be the orthogonal projections onto the subspaces $A_{n+1}$ and $B_{n}$ of $B_{n+1}$. Then, the commuting square condition says that $e_{A_{n+1}} e_{B_{n}}=e_{A_{n}}=e_{B_{n}} e_{A_{n+1}}$ for all $n \in \mathbb{N}$. Finally, we also note that the trace tr is uniquely determined on $A_{n}$ and $B_{n}$ by its weight vectors $\boldsymbol{a}_{n}$ and $\boldsymbol{b}_{n}$ which are defined as follows: Let $p_{\mu}$ be a minimal idempotent in the simple component of $A_{n}$ labeled by $\mu$. Then we define $a_{n, \mu}=\operatorname{tr}\left(p_{\mu}\right)$, and $\boldsymbol{a}_{n}=\left(a_{n, \mu}\right)_{\mu}$, where $\mu$ runs through a labeling set of the simple components of $A_{n}$. The weight vector $\boldsymbol{b}_{n}$ for $B_{n}$ is defined similarly. The following proposition follows from [ibid.], Theorem 1.5 (where the matrix $G=\left(g_{\lambda \mu}\right)$ defined here would correspond to the matrix $G^{t}$ in [ibid.]).

Proposition 1.1. Under the given conditions, we get a subfactor $\mathcal{N} \subset \mathcal{M}$ whose index $[\mathcal{M}: \mathcal{N}]$ is equal to $\left\|\boldsymbol{a}_{n}\right\|^{2} /\left\|\boldsymbol{b}_{n}\right\|^{2}$ for any sufficiently large $n$. Moreover, we have $\sum g_{\lambda \mu} a_{n, \lambda}=[\mathcal{M}: \mathcal{N}] b_{n, \mu}$.

1B. Special periodic algebras. In general, it can be quite hard to determine finer invariants of the subfactors, the so-called higher relative commutants (or centralizers) from the generating sequence of algebras. However, under certain circumstances, this can become quite easy. We describe such a setup. It is a moderate abstraction of an approach which has already been used before by a number of authors. The reader familiar with tensor categories and module categories should think of the algebras $A_{n}=\operatorname{End}_{\mathscr{C}}\left(X^{\otimes n}\right)$ and $B_{n}=\operatorname{End}_{\mathscr{D}}\left(Y \otimes X^{\otimes n}\right)$ for $X$ an object in a $C^{*}$ tensor 
category $\mathscr{C}$ and $Y$ an object in a module category $\mathscr{D}$ over $\mathscr{C}$. In the following, we will make the following assumptions beyond the ones in the previous subsection:

1. The algebras $A_{n}$ will be monoidal $C^{*}$-algebras. This means we have canonical embeddings of $C^{*}$ algebras $A_{m} \otimes A_{n} \rightarrow A_{n+m}$ with multiplicativity of the trace, that is, $\operatorname{tr}\left(a_{1} \otimes a_{2}\right)=\operatorname{tr}\left(a_{1}\right) \operatorname{tr}\left(a_{2}\right)$.

2. We have canonical embeddings $B_{m} \otimes A_{n} \rightarrow B_{n+m}$, again with multiplicativity of the trace.

3. We have the commuting square condition for the sequences of algebras $A_{n} \subset B_{n}$ and $1 \otimes A_{n-1} \subset A_{n}$.

4. There exists $d \in \mathbb{N}$ and a projection $p \in A_{d}$ such that $\left(1_{m} \otimes p\right) A_{m+d}(1 \otimes p) \cong A_{m}$ and $\left(1_{m} \otimes p\right) B_{m+d}(1 \otimes p) \cong B_{m}$ for all $m \in \mathbb{N}$.

Examples for this setup will be given at the end of this section and in Section 2. Moreover, any finite depth subfactor $\mathcal{N} \subset \mathcal{M}$ (see [Goodman et al. 1989; Evans and Kawahigashi 1998] for definitions) produces algebras for such a setup as follows: Let $\mathcal{M}^{\otimes n}=\mathcal{M} \otimes_{\mathcal{N}} \mathcal{M} \otimes_{\mathcal{N}} \cdots \otimes_{\mathcal{N}} \mathcal{M}$ ( $n$ factors). Obviously, $\mathcal{M}^{\otimes n}$ is an $\mathcal{N}-\mathcal{N}$ as well as an $M-\mathcal{N}$ bimodule. One can check that for

$$
A_{n}=\operatorname{End}_{\mathcal{N}-\mathcal{N}} M^{\otimes n} \subset B_{n}=\operatorname{End}_{\mathcal{M}-\mathcal{N}} \mathcal{M}^{\otimes n+1}
$$

the axioms above are satisfied; here the embedding is defined by letting the elements of $A_{n}$ act on the second to $(n+1)-s t$ factor of $\mathcal{M}^{\otimes n+1}$. It is also possible to define these algebras in connection of relative commutants in the Jones tower of relative commutants (see [Bisch 1997] for details). Recall that for factors $\mathcal{N} \subset \mathcal{M}$ the relative commutant (or centralizer) $\mathcal{N}^{\prime} \cap \mathcal{M}$ is defined to be the set $\{b \in \mathcal{M} \mid a b=b a$ for all $a \in \mathcal{N}\}$.

Lemma 1.2. The subfactor $\mathcal{N} \subset \mathcal{M}$ generated from the sequences of algebras $1_{m} \otimes A_{n} \subset B_{n+m}$ has relative commutant $B_{m}$. The same statement also holds with $B_{n+m}$ and $B_{m}$ in the last sentence replaced by $A_{n+m}$ and $A_{m}$.

Proof. This is essentially the proof used for Theorem 3.7 in [Wenzl 1988a]. Observe that by induction on $r$ and assumption 4 above, we also have

$$
\left(1_{m} \otimes p^{\otimes r}\right) X_{m+r d}\left(1_{m} \otimes p^{\otimes r}\right) \cong X_{m}
$$

for $X=A, B$. It follows from Theorem 1.6 of [ibid.] that the dimension of the relative commutant $\mathcal{N}^{\prime} \cap \mathcal{M}$ is at most equal to the dimension of $B_{m}$. The claim follows from the fact that $B_{m} \otimes 1_{n}$ commutes with $1_{m} \otimes A_{n}$ for all $n$.

1C. Bimodules and principal graphs. We calculate the first principal graph for subfactors constructed in our setup, using fairly elementary methods from [ibid.] as well as the bimodule approach. The latter was first used in the subfactor context 
by Ocneanu; see [Evans and Kawahigashi 1998]. For the connection between bimodules and principal graphs, see [Bisch 1997] and for more details compatible with our notation, see also [Erlijman and Wenzl 2007]. While most of this section has already appeared before implicitly or explicitly, the presentation in our setup might be useful also in other contexts.

Pick $k$ large enough so that $m=k d>n_{0}$. Hence, the inclusion matrices for $A_{r d} \subset B_{r d}$ coincide for all $r \geq k$ using the bijection of simple components as described in Section 1A. Let $\Lambda_{m}$ and $\tilde{\Lambda}_{m}$ be labeling sets for the simple components of $B_{m}$ and $A_{m}$ respectively. Let $\mathcal{N}$ and $\mathcal{M}$ be the factors generated by the increasing sequences of algebras $A_{n}$ and $B_{n}$ respectively; see Proposition 1.1 or Lemma 1.2, with the $m$ there equal 0 . Both of these factors have a subfactor $\tilde{\mathcal{N}}$ generated by the subalgebras

$$
1_{m} \otimes A_{n} \subset A_{n+m} \subset B_{n+m} .
$$

We now define for each $\lambda \in \tilde{\Lambda}_{m}$ an $\mathcal{N}-\tilde{\mathcal{N}}$ bimodule $N_{\lambda}$ as follows: It is the Hilbert space completion of $\mathcal{N} p_{\lambda}$ with respect to the inner product induced by tr, where $p_{\lambda}$ is a minimal idempotent in $A_{m, \lambda}$, the simple component of $A_{m}$ labeled by $\lambda$, with obvious left and right actions by $\mathcal{N}$ and $\tilde{N}$. To ease notation, we shall often refer to it as an $\mathcal{N}-\mathcal{N}$ bimodule, using the isomorphism between $\tilde{\mathcal{N}}$ and $\mathcal{N}$ given by the trace preserving maps $a \in A_{n} \mapsto 1_{m} \otimes a \in A_{n+m}$.

Similarly, we define $\mu-\tilde{\mathcal{N}}$ bimodules $M_{\mu}$ for any $\mu \in \Lambda_{m}$ which are Hilbert space completions of $M p_{\mu}$, where $p_{\mu}$ is a minimal idempotent in the simple component $B_{m, \mu}$ of $B_{m}$. Finally, we define the inclusion numbers $b_{\mu}^{\lambda}$ for elements $\lambda \in \tilde{\Lambda}$ and $\mu \in \Lambda_{m}$ as usual (see Section 1A).

Lemma 1.3. The bimodules $N_{\lambda}$ and $M_{\mu}$ are irreducible $\mathcal{N}-\tilde{\mathcal{N}}$ and $\mu-\tilde{\mathcal{N}}$ bimodules, respectively. We have the decomposition $M_{\mu} \cong \bigoplus_{\lambda} b_{\mu}^{\lambda} N_{\lambda}$ as $\mathcal{N}-\tilde{\mathcal{N}}$ modules.

Proof. This is well-known (see [Erlijman and Wenzl 2007] for more details). It follows from Lemma 1.2 that the endomorphism ring of the $M-\tilde{\mathcal{N}}$ bimodule $M$ is given by $B_{m}$. Hence the $\mu-\tilde{\mathcal{N}}$ bimodules $M_{\mu}$ are simple, as $p_{\mu}$ was chosen to be a minimal idempotent in $B_{m}$. One shows similarly that also the $N_{\lambda}$ are simple $\mathcal{N}-\tilde{\mathcal{N}}$ bimodules.

Observe that $\operatorname{dim}_{\mathcal{N}} N_{\lambda}=\operatorname{tr}\left(p_{\lambda}\right)$ and $\operatorname{dim}_{\mathcal{M}} M_{\mu}=\operatorname{tr}\left(p_{\mu}\right)$; see [Jones 1983]. Now if $p_{\lambda}$ is a minimal idempotent in $A_{m}$, it follows from the definitions that $\operatorname{Ind}_{\mathcal{N}}^{\mathcal{M}} N_{\lambda}:=$ $M p_{\lambda}$ is isomorphic as an $M-\tilde{\mathcal{N}}$ bimodule to the direct sum $\oplus b_{\mu}^{\lambda} M_{\mu}$. By Frobenius reciprocity (see [Evans and Kawahigashi 1998; Bisch 1997]) it follows that the module $N_{\lambda}$ appears with multiplicity $b_{\mu}^{\lambda}$ in $\mu_{\mu}$, viewed as an $\mathcal{N}-\tilde{\mathcal{N}}$ bimodule. Hence the $\mathcal{N}-\tilde{\mathcal{N}}$ bimodule $M_{\mu}$ has a submodule which is isomorphic to $\bigoplus_{\lambda} b_{\mu}^{\lambda} N_{\lambda}$. But as $M_{\mu}$ has $\mathcal{N}$-dimension $[\mathcal{M}: \mathcal{N}] \operatorname{tr}\left(p_{\mu}\right)$, it coincides with this submodule, by Proposition 1.1. 
Theorem 1.4. Let $\mathcal{N} \subset \mathcal{M}$ be the subfactor generated by sequences of algebras $A_{n} \subset B_{n}$ satisfying the conditions in Section 1B. Then its first principal graph is given by the inclusion graph for $A_{k d} \subset B_{k d}$ for sufficiently large $k$.

Proof. It is well-known that the first principal graph is given by the inductionrestriction graph of $\mathcal{M}-\mathcal{N}$ and $\mathcal{N}-\mathcal{N}$ bimodules appearing in the tensor products $\mathcal{M}^{\otimes n}, n \in \mathbb{N}$, where $\mathcal{M}^{\otimes n}=\mathcal{M} \otimes_{\mathcal{N}} \mathcal{M} \otimes_{\mathcal{N}} \cdots \otimes_{\mathcal{N}} \mathcal{M}$ ( $n$ factors); see [Evans and Kawahigashi 1998; Bisch 1997]. Obviously, this graph does not change if we replace all $X-\mathcal{N}$ bimodules $H$ in this setting by $X-q \mathcal{N} q$ bimodules $H q$, for $X=\mathcal{M}, \mathcal{N}$ and $q$ a nonzero projection in $\mathcal{N}$. The claim can now be shown for $q=p^{\otimes k}$ where $k$ is chosen large enough so that $k d>n_{0}$, using Lemma 1.3.

Recall that many examples come from module tensor categories, where $A_{n}=$ $\operatorname{End}_{\mathbb{C}}\left(X^{\otimes n}\right)$ and $B_{n}=\operatorname{End}\left(Y \otimes\left(X^{\otimes n}\right)\right)$ for an object $X$ in a tensor category $\mathscr{C}$ and an object $Y$ in the module category $\mathscr{D}$ over $\mathscr{C}$. In this setting, the weight vectors of our trace are given by $a_{n, \lambda}=\tilde{d}_{\lambda} / x^{n}$ and $b_{n, \mu}=d_{\mu} / y x^{n}$ for positive quantities $d_{\mu}, \tilde{d}_{\lambda}, x$ and $y$. Then we have:

Corollary 1.5. Assuming the conditions for the trace weights as just given, we have subfactors $\mathcal{N} \subset \mathcal{M}_{\mu}$ with index $\left[\mathcal{M}_{\mu}: \mathcal{N}\right]=d_{\mu}^{2}[\mathcal{M}: \mathcal{N}]$, with $\mathcal{N} \subset \mathcal{M}$ as in Theorem 1.4.

Remark 1.6. There is also a second important invariant for $\mathcal{N} \subset \mathcal{M}$, the dual principal graph. It can be analogously defined as an induction-restriction graph between irreducible $\mathcal{M}-\mathcal{M}$ and $\mathcal{M}-\mathcal{N}$ bimodules appearing in the tensor powers $\mathcal{M}^{\otimes n}$. Its calculation is more difficult than that of the first principal graph. This is quite similar to the corresponding problem for subfactors coming from conformal inclusions and related constructions; see [Xu 1998; Böckenhauer et al. 1999; Erlijman and Wenzl 2007]. We plan to study this problem in a future publication via suitable adaptions of techniques in those papers.

1D. The GHJ-construction. We give a well-known and well-studied example for our current setup, which was first constructed in [Goodman et al. 1989]. Let $G$ be a matrix with nonnegative integer entries and norm less than 2. It is well-known that such matrices are classified by Coxeter graphs of type $A D E$. We assume that the columns of $G$ are indexed by the even vertices, and the rows by the odd vertices. We define $C^{*}$-algebras $B_{n}$ by $B_{0}=\mathbb{C}^{v_{e}}$, and $B_{1}=\oplus M_{d_{j}}$, where $v_{e}$ is the number of even vertices, and the summands of $B_{1}$ are labeled by the odd vertices $j$, whose dimension $d_{j}$ is equal to the number of even vertices to which $j$ is connected. The embedding $B_{o} \subset B_{1}$ is given by the inclusion matrix $G$. Then we define recursively $B_{n+1}$ via Jones' basic construction [1983] for $B_{n-1} \subset B_{n}$. Here the trace on $B_{n}$ is the unique normalized trace whose values on minimal idempotents are given by the Perron-Frobenius vector of $G^{t} G$ or $G G^{t}$, depending on whether $n$ is even or odd, and the vector is normalized such that $\operatorname{tr}(1)=1$. Then the algebra $B_{n+1}$ is generated 
by $B_{n}$, acting on itself via left multiplication, and the orthogonal projection $e_{n}$ onto the subspace $B_{n-1}$ of $B_{n}$, with respect to the inner product coming from the trace. The algebra $A_{n}$ is defined to be the subalgebra generated by the identity 1 and the projections $e_{i}, 1 \leq i<1$. It is well-known that these algebras satisfy the commuting square condition, that they are periodic with periodicity 2 , and that the Jones projections $e_{i}$ satisfy the conditions of the projection $p$ in Section 1B. This has already been shown in [Goodman et al. 1989].

\section{2. $q$-Brauer algebras}

2A. Definitions. Fix $N \in \mathbb{Z}$ and let $[N]=\left(q^{N}-q^{-N}\right) /\left(q-q^{-1}\right)$, where $q$ is considered to be a complex number. We denote by $H_{n}\left(q^{2}\right)$ the Hecke algebra of type $A_{n-1}$. It is given by generators $g_{1}, g_{2}, \ldots, g_{n-1}$ which satisfy the usual braid relations and the quadratic relation $g_{i}^{2}=\left(q^{2}-1\right) g_{i}+q^{2}$. The $q$-Brauer algebra $\operatorname{Br}_{n}(N)$ is the complex algebra defined via generators $g_{1}, g_{2}, \ldots, g_{n-1}$ and $e$ and the following relations:

(H) $g_{1}, g_{2}, \ldots, g_{n-1}$ satisfy the relations of the Hecke algebra $H_{n}\left(q^{2}\right)$.

(E1) $e^{2}=[N] e$.

(E2) $e g_{i}=g_{i} e$ for $i>2, e g_{1}=q^{2} e, e g_{2} e=q^{N+1} e$ and $e g_{2}^{-1} e=q^{-1-N} e$.

(E3) $g_{2} g_{3} g_{1}^{-1} g_{2}^{-1} e_{(2)}=e_{(2)}=e_{(2)} g_{2} g_{3} g_{1}^{-1} g_{2}^{-1}$, where $e_{(2)}=e\left(g_{2} g_{3} g_{1}^{-1} g_{2}^{-1}\right) e$.

Remark 2.1. (a) Relation (E3) can be replaced by the perhaps slightly less mysterious relation $e_{(2)} g_{2} g_{3}=e_{(2)} g_{2} g_{1}$ and $g_{1}^{-1} g_{2}^{-1} e_{(2)}=g_{3}^{-1} g_{2}^{-1} e_{(2)}$.

(b) It is easy to see that this algebra coincides with the algebra defined in [Wenzl 2012] after substituting $q$ there by $q^{2}$, and $e$ there by $q^{1-N} e$ (with the $q$ of this paper); this is also compatible with the different definition of $[N]$ in [ibid.]. We have chosen this parametrization as it will make it easier to define a $*$-structure on it. More precisely, if $|q|=1$, there exists a complex conjugate antiautomorphism $b \mapsto b^{*}$ on $\mathrm{Br}_{n}(N)$ defined by

$$
e^{*}=e, \quad g_{i}^{*}=g_{i}^{-1}, \quad \text { where } 1 \leq i<n .
$$

It is easy to check the relations to show this operation is well-defined.

2B. Molev representation. We give a representation of our algebra $\operatorname{Br}_{n}(N)$ in $\operatorname{End}\left(V^{\otimes n}\right)$, where $V=\mathbb{C}^{N}$. For this we use the matrices used by Molev [2003] for the definition of his $q$-deformation of Brauer's centralizer algebra. His defining relations are slightly different from ours, but Molev has informed the author that our algebra satisfies the relations of his algebra. It turns out that also his matrices satisfy the relations of our algebras, which we will outline here. Let $R$ be the well-known solution of the quantum Yang-Baxter equation for type $A$. For simplicity we will 
use this notation for what is often denoted as $\check{R}$. If $E_{i j}$ are the matrix units for $n \times n$ matrices, we define the following elements in $\operatorname{End}\left(V^{\otimes 2}\right)$ :

$$
R=\sum_{i} q E_{i i} \otimes E_{i i}+\sum_{i \neq j} E_{i j} \otimes E_{j i}+\sum_{i<j}\left(q-q^{-1}\right) E_{i i} \otimes E_{j j},
$$

and

$$
Q=\sum_{i, j} q^{N+1-2 i} E_{i j} \otimes E_{i j}
$$

Moreover, if $A \in \operatorname{End}\left(V^{\otimes 2}\right)$, we define the operator $A_{i} \in \operatorname{End}\left(V^{\otimes n}\right)$ by

$$
A_{i}=1_{i-1} \otimes A \otimes 1_{n-1-i},
$$

where $1_{k}$ is the identity on $V^{\otimes k}$. Then we have the following proposition, all of whose essential parts were already proved in [Molev 2003]. However, the relations for our algebras are slightly different, so we spell out some of the adjustments of the work in [ibid.] in our context below.

Proposition 2.2. The map $g_{i} \mapsto q R_{n-i}$ and $e \mapsto Q_{n-1}$ defines a representation $\Phi$ of $\operatorname{Br}_{n}(N)$. It specializes to the usual representation of Brauer's centralizer algebra in $\operatorname{End}\left(V^{\otimes n}\right)$ for $q=1$.

Proof. Most of the relations are already known or are easy to check. For example, it is well-known that the matrices $q R_{i}$ satisfy the relations of the Hecke algebra $H_{n}\left(q^{2}\right)$. Relation (E1) is checked easily, and also the relations in (E2) are fairly straightforward to check. It suffices to check (E3) for $n=4$. For this observe that by [ibid., (4.16)], we have

$$
Q_{3} R_{2} R_{3} R_{1} R_{2} Q_{3}=Q_{1} Q_{3}+q^{N+1}\left(q-q^{-1}\right) Q_{3}\left(R_{1}+q^{-1} 1\right),
$$

in our notation. Using the relation $R_{i}=R_{i}^{-1}+\left(q-q^{-1}\right) 1$ for the second and third factor of the left hand side, one derives from this

$$
Q_{3} R_{2}^{-1} R_{3}^{-1} R_{1} R_{2} Q_{3}=Q_{1} Q_{3} .
$$

To check relation (E3), observe that

$$
R_{1} R_{2}\left(v_{i} \otimes v_{i} \otimes v_{j} \otimes v_{j}\right)=R_{3} R_{2}\left(v_{i} \otimes v_{i} \otimes v_{j} \otimes v_{j}\right),
$$

where $\left(v_{i}\right)$ is the standard basis for $\mathbb{C}^{N}=V$. One derives from this that

$$
R_{2}^{-1} R_{1}^{-1} R_{3} R_{2} Q_{1} Q_{3}=Q_{1} Q_{3} .
$$

Moreover, the same calculations above also work with $R_{i}$ replaced by $R_{i}^{-1}$ and $Q_{j}$ replaced by its transpose $Q_{j}^{T}$. Hence one can show as before that

$$
R_{2} R_{3} R_{1}^{-1} R_{2}^{-1} Q_{1}^{T} Q_{3}^{T}=Q_{1}^{T} Q_{3}^{T} .
$$


Transposing this, using $R_{i}^{T}=R_{i}$ shows the last part of the claim.

2C. Quotients. We can now rephrase the main results of [Wenzl 2012] in our notation as follows:

Theorem 2.3. (a) There exists a well-defined functional tr on $\operatorname{Br}_{n}(N)$ defined inductively by $\operatorname{tr}\left(g_{1}\right)=q^{N+1} /[N], \operatorname{tr}(e)=1 /[N]$ and $\operatorname{tr}\left(b g_{n}\right)=\operatorname{tr}(b) \operatorname{tr}\left(g_{n}\right)$ for all $b \in \mathrm{Br}_{n}(N)$.

(b) Let $\overline{\mathrm{Br}}_{n}(N)=\mathrm{Br}_{n}(N) / I_{n}$, where $I_{n}$ is the annihilator ideal of tr. Then $\overline{\mathrm{Br}}_{n}(N)$ is semisimple and the inclusion $\overline{\mathrm{Br}}_{n}(N) \subset \overline{\mathrm{Br}}_{n+1}(N)$ is well-defined for all $n$.

It is possible to explicitly describe the structure of the quotients $\overline{\mathrm{Br}}_{n}=\overline{\mathrm{Br}}_{n}(N)$. To do so, we need the following definitions for the labeling sets of simple representations. More conceptually, the labeling sets $\Lambda(N, \ell)$ consist of all such diagrams $\lambda$ for which the quantities $d_{\mu}(q) \neq 0$ for any subdiagrams $\mu \subset \lambda$ including $\lambda$ itself, where $q^{2}$ is a primitive $\ell$-th root of unity and the $d_{\mu}$ are defined in Section 2E.

Definition 2.4. Fix integers $N$ and $\ell$ satisfying $1<|N|<\ell$.

(i) The set $\tilde{\Lambda}(N, \ell)$ consists of all Young diagrams with at most $N$ rows such that the first and $N$-th row differ by at most $\ell-N$ boxes for $N>0$. If $N<0$, the Young diagrams have at most $|N|$ columns, where the first and $|N|$-th column differ by at most $\ell-|N|$ boxes.

(ii) The set $\Lambda(N, \ell)$ consists of all Young diagrams $\lambda$ with $\lambda_{i}$ boxes in the $i$-th row and $\lambda_{j}^{\prime}$ boxes in the $j$-th column which satisfy

(a) $\lambda_{1}^{\prime}+\lambda_{2}^{\prime} \leq N$ and $\lambda_{1} \leq(\ell-N) / 2$ if $N>0$ and $\ell-N$ even,

(b) $\lambda_{1}^{\prime}+\lambda_{2}^{\prime} \leq N$ and $\lambda_{1}+\lambda_{2} \leq \ell-N$ if $N>0$ and $\ell-N$ odd,

(c) $\lambda_{1} \leq|N| / 2$ and $\lambda_{1}^{\prime}+\lambda_{2}^{\prime} \leq \ell-|N|$ if $N<0$ is even,

(d) $\lambda_{1}+\lambda_{2} \leq|N|$ and $\lambda_{1}^{\prime}+\lambda_{2}^{\prime} \leq \ell-|N|$ if $N<0$ is odd.

Diagrams which miss one of these inequalities only by the quantity one are called boundary diagrams of $\Lambda(N, \ell)$; for example in case (a) if $\lambda_{1}^{\prime}+\lambda_{2}^{\prime}=N+1$.

Theorem 2.5 [Wenzl 2012, Section 5]. Let $q^{2}$ be a primitive $\ell$-th root of unity, and let $N$ be an integer satisfying $1<|N|<\ell$. Then the simple components of $\overline{\mathrm{Br}}_{n}=\overline{\mathrm{Br}}_{n}(N)$ are labeled by the Young diagrams in $\Lambda(N, \ell)$ with $n, n-2, n-4, \ldots$ boxes. If $V_{n, \lambda}$ is a simple $\overline{\mathrm{Br}}_{n}$-module for such a diagram $\lambda$, it decomposes as a $\mathrm{Br}_{n-1}$ module as

$$
V_{n, \lambda} \cong \bigoplus_{\mu} V_{n-1, \mu}
$$

where $\mu$ runs through diagrams in $\Lambda(N, \ell)$ obtained by removing or, if $|\lambda|<n$, also by adding a box to $\lambda$. 
2D. Path idempotents and matrix units. We will give some details about the proof of Theorem 2.5 which will also be needed for further results. Observe that the restriction rule (2-2) implies that a minimal idempotent $p_{\mu}$ in $\overline{\mathrm{Br}}_{n-1, \mu}$ can be written as a sum of minimal idempotents with exactly one in $\overline{\mathrm{Br}}_{n, \lambda}$ for each diagram $\lambda$ in $\Lambda(N, \ell)$ that can be obtained by adding or subtracting a box from $\mu$. This inductively determines a system of minimal idempotents and matrix units of $\overline{\mathrm{Br}}_{n}\left(q^{N}, q\right)$ labeled by paths and pairs of paths, respectively, in $\Lambda(N, \ell)$ of length $n$. Such a path is defined to be a sequence of Young diagrams $\left(\lambda^{(i)}\right)_{i=0}^{n}$ where $\lambda^{(0)}$ is the empty Young diagram, and $\lambda^{(i+1)}$ is obtained from $\lambda^{(i)}$ by adding or removing a box. It follows from the restriction rule above that the dimension of $V_{n, \lambda}$ is equal to the number of paths of length $n$ with $\lambda^{(n)}=\lambda$, and that we can label a complete system of matrix units for the simple component $\overline{\mathrm{Br}}_{n, \lambda}$ by pairs of such paths. We then have the following lemma:

Lemma 2.6. For each pair of paths $t_{1}, t_{2}$ in $\Lambda(N, \infty)$ with the same endpoint we can define the matrix unit $E_{t_{1}, t_{2}}$ as a linear combination of products of generators over algebraic functions (rational for path idempotents) in $q$ with poles only at roots of unity. More precisely, the formula for $E_{t_{1}, t_{2}}$ is well-defined for $q^{2}$ a primitive $\ell$-th root of unity if both $t_{1}$ and $t_{2}$ are paths in $\Lambda(N, \ell)$.

Proof. This was proved in [Wenzl 2012], Section 5. As the result is not explicitly stated as such, we give some details here. One observes that the two-sided ideal generated by the element $\bar{e} \in \overline{\mathrm{Br}}_{n+1}$ is isomorphic to Jones' basic construction for the algebras $\overline{\mathrm{Br}}_{n-1} \subset \overline{\mathrm{Br}}_{n}$ (or, strictly speaking, by certain conjugated subalgebras which are denoted by $i_{1}\left(\overline{\mathrm{Br}}_{n}\right)$ and $i_{2}\left(\overline{\mathrm{Br}}_{n-1}\right)$; see Section 5.2 in [Wenzl 2012]). One can then define path idempotents and matrix units inductively as it was done in [Ram and Wenzl 1992, Theorem 1.4], using the formulas for the weights of traces, which will also be reviewed in Section 2E; this is closely related to what is also known in subfactor theory as the Ocneanu-Sunder path model [Sunder 1987]. The complement of this ideal is a quotient of the Hecke algebra $\bar{H}_{n+1}$, for which matrix units already were more or less defined in [Wenzl 1988a, p. 366].

Lemma 2.7. Let $p_{[N]}$ and $p_{\left[1^{N}\right]}$ be the minimal idempotents in $H_{N}$ corresponding to its one-dimensional trivial and sign representations. Then for all $m>0$ we have

$$
\bar{p}_{\left[1^{N}\right]}^{\otimes 2} \overline{\mathrm{Br}}_{m+2 N} \bar{p}_{\left[1^{N}\right]}^{\otimes 2} \cong \overline{\mathrm{Br}}_{m}
$$

for $N>0$, and

$$
p_{[-N]} \overline{\mathrm{Br}}_{-N+m} p_{[-N]} \cong \overline{\mathrm{Br}}_{m}
$$

for $N<0$ even.

Proof. Let us assume $N>0$ first. Observe that if $p \in \overline{\mathrm{Br}}_{2 N, \varnothing}(N)$, the simple component labeled by the empty Young diagram $\varnothing$, then it follows from the restriction 
rule (2-2) (see also the equivalent version below Theorem 2.5) by induction on $m$ that $p \overline{\mathrm{Br}}_{m+2 N}(N) p \cong \overline{\mathrm{Br}}_{m}(N)$ for all $m \geq 0$. Hence it suffices to show that $p_{\left[1^{N}\right]}^{\otimes 2}$ is such an idempotent.

If $q=1$ and $N>0, \Phi\left(\mathrm{Br}_{n}(N)\right)$ coincides with the commutant of the action of the orthogonal group $\mathrm{O}(N)$ on $V^{\otimes n}$, which is semisimple. Moreover, the trace tr is just a multiple of the pull-back of the natural trace on $\operatorname{End}\left(V^{\otimes n}\right)$, so $\Phi\left(\operatorname{Br}_{n}(N)\right) \cong \overline{\mathrm{Br}}_{n}(N)$ at $q=1$. As $\Phi\left(p_{\left[1^{N}\right]}\right)$ projects onto the one-dimensional determinant representation in $V^{\otimes N}$, the claim follows easily in that case, using Brauer duality, that is, the fact that $\Phi\left(\operatorname{Br}_{n}(N)\right)$ is equal to the commutant of $\mathrm{O}(N)$ on $V^{\otimes n}$ for all $n$.

We will now use the fact that we can also define $\operatorname{Br}_{n}(N)$ over the field of rational functions $\mathbb{C}(q)$; see [Wenzl 2012]. It follows from Lemma 2.6 that we can also define the path idempotents for $\overline{\mathrm{Br}}_{n}(N)$ over that field for paths of length $n$ in $\Lambda(N, \infty)$. As the rank of an idempotent is an integer, the claim follows as well for $q$ a variable, and for $q \in \mathbb{C}$ not a root of unity. But as $p_{t} \bar{p}_{\left[1^{N}\right]}^{\otimes 2} p_{t}=0$ for any path $t$ of length $2 N$ in $\Lambda(N, \ell)$ which ends in $\lambda \neq \varnothing$, we also get rank 0 for $\bar{p}_{\left[1^{N}\right]}^{\otimes 2}$ at $q^{2}$ a primitive $\ell$-th root of unity in $\overline{\mathrm{Br}}_{2 N, \lambda}(N)$. One also shows by a similar continuity and path idempotent argument that the rank of $p_{\left[1^{N}\right]}^{\otimes 2}$ is equal to 1 in $\overline{\mathrm{Br}}_{2 N, \varnothing}(2 n)$. This finishes the proof for $N>0$.

If $N<0$ even, we would map the permutation $(i, i+1)$ to the negative of the linear map permuting the $i$-th and $(i+1)$-st factor of $V^{\otimes n}$, where $V=\mathbb{C}^{|N|}$. Hence $p_{[-N]}$ would map onto the antisymmetrization of $V^{\otimes-N}$, on which $\operatorname{Sp}(|N|)$ acts trivially. The map above extends to a map of $\operatorname{Br}_{n}(N)$ onto $\operatorname{End}_{\operatorname{Sp}(|N|}\left(V^{\otimes n}\right)$ (see [Wenzl 1988b]), and we can now duplicate the proof for the orthogonal case.

2E. Weights of the trace. Using the character formulas of orthogonal groups, one can calculate the weights of $\operatorname{tr}$ for the algebras $\operatorname{Br}_{n}(N)$, that is, its values at minimal idempotents of $\operatorname{Br}_{n}(N)$. We will need the following quantities for a given Young diagram $\lambda$ :

$$
d(i, j)= \begin{cases}\lambda_{i}+\lambda_{j}-i-j & \text { if } i \leq j, \\ -\lambda_{i}^{\prime}-\lambda_{j}^{\prime}+i+j-2 & \text { if } i>j .\end{cases}
$$

Moreover, we define $h(i, j)$ to be the length of the hook in the Young diagram $\lambda$ whose corner is the box in the $i$-th row and $j$-th column. We can now restate [Wenzl 2012], Theorem 4.6 in the notations of this paper as follows:

Theorem 2.8. The weights of the Markov trace tr for the Hecke algebra $\bar{H}_{n}\left(q^{2}\right)$ are given by $\tilde{\omega}_{\lambda}=\tilde{d}_{\lambda} /[N]^{n}$, where $|\lambda|=n$, and for $\overline{\mathrm{Br}}_{n}(N)$ they are given by $\omega_{\lambda, n}=d_{\lambda} /[N]^{n}$, where

$$
\tilde{d}_{\lambda}=\prod_{(i, j) \in \lambda} \frac{[N+j-i]}{[h(i, j)]}, \quad d_{\lambda}=\prod_{(i, j) \in \lambda} \frac{[N+d(i, j)]}{[h(i, j)]},
$$


where $\lambda$ runs through all the Young diagrams in $\tilde{\Lambda}(N, \ell)$ with $n$ boxes for $\bar{H}_{n}\left(q^{2}\right)$, and through all Young diagrams in $\Lambda(N, \ell)$ with $n, n-2, n-4, \ldots$ boxes for $\overline{\mathrm{Br}}_{n}$.

Lemma 2.9. The weights $\omega_{\lambda, n}$ are positive for all $\lambda \in \Lambda(N, \ell)$ if and only if $q^{2}=e^{ \pm 2 \pi i / \ell}$ with $\ell>N$ and

(a) $N>0$ and $\ell-N$ even, or

(b) $N<0$ odd.

Proof. The weights can be rewritten for our choice of $q$ as

$$
\omega_{\lambda, n}=\frac{\sin ^{n}(\pi / \ell)}{\sin ^{n}(N \pi / \ell)^{n}} \prod_{(i, j) \in \lambda} \frac{\sin (N+d(i, j)) \pi / \ell}{\sin (h(i, j) \pi / \ell)} .
$$

As $h(i, j) \leq h(1,1)=\lambda_{1}+\lambda_{1}^{\prime}-1<\ell$ for all boxes $(i, j)$ of $\lambda$, it follows that all factors in the formula above are positive for $N>0$ (negative for $N<0$ ) except possibly the ones in the numerator under the product. If $N>0$ and $\ell-N$ odd, one checks that for the diagram $[\ell-N+1) / 2]$ we have $\omega_{\lambda,|\lambda|}<0$. By the same argument, one shows that $\omega_{\lambda,|\lambda|}<0$ for $\lambda=[(|N|+1) / 2]$ and $N<0$. In the other two cases, one checks that $0<|d(i, j)|<\ell$ for all boxes $(i, j)$ of a diagram $\lambda \in \Lambda(N, \ell)$.

\section{F. $C^{*}$-quotients.}

Proposition 2.10. If the weights $\omega_{\lambda, n}$ are positive for all $\lambda \in \Lambda(N, \ell)$, the star operation defined by $e^{*}=e$ and by $g_{i}^{*}=g_{i}^{-1}$ makes the quotients $\overline{\mathrm{Br}}_{n}$ into $C^{*}$-algebras.

Proof. The proof goes by induction on $n$, with the claims for $n=1$ and $n=2$ easy to check. By [Wenzl 2012], the two-sided ideal $I_{n+1}$ generated by $e$ in $\overline{\mathrm{Br}}_{n+1}$ is isomorphic to Jones' basic construction for $\overline{\mathrm{Br}}_{n-1} \subset \overline{\mathrm{Br}}_{n}$; see also the remarks before Lemma 2.7. In particular, $I_{n+1}$ is spanned by elements $b_{1} e b_{2}$, with $b_{1}, b_{2} \in i_{1}\left(\overline{\mathrm{Br}}_{n}\right)$, where $i_{1}(a)=\Delta_{n+1} a \Delta_{n+1}^{-1}$, with $\Delta=\left(g_{1} g_{2} \cdots g_{n-1}\right)\left(g_{1} \cdots g_{n-2}\right) \cdots g_{1}$. By induction assumption and properties of Jones' basic construction, this ideal has a $C^{*}$-structure given by $\left(b_{1} e b_{2}\right)^{*}=b_{2}^{*} e b_{1}^{*}$. This coincides with the $*$ operation defined before algebraically. It was shown in [Wenzl 2012] that $\overline{\mathrm{Br}}_{n+1} \cong I_{n+1} \oplus \bar{H}_{n+1}$, where $\bar{H}_{n+1}$ is a semisimple quotient of the Hecke algebra $H_{n+1}$ whose simple components are labeled by the Young diagrams $\lambda \in \Lambda(N, \ell)$ with $n+1$ boxes. All these simple representations satisfy the $(k, \ell)$ condition in [Wenzl 1988a]. It follows from that paper that the map $g_{i}^{*}=g_{i}^{-1}$ induces a $C^{*}$ structure for any such representation. This finishes the proof.

Theorem 2.11. For each choice of $N$ and $\ell$ with $q^{2}=e^{ \pm 2 \pi i / \ell}$, and for each nonnegative integer $m$, we obtain a subfactor $\mathcal{N} \subset \mathcal{M}$ with $\mathcal{N}^{\prime} \cap \mathcal{M}=\overline{\mathrm{Br}}_{m}$ and with index

$$
[\mathcal{M}: \mathcal{N}]=[N]^{m} \frac{\sum_{\mu \in \tilde{\Lambda}(N, \ell),|\mu|=k|N|} \tilde{d}_{\mu}^{2}}{\sum_{\lambda \in \Lambda(N, \ell), 2|| \lambda \mid} d_{\lambda}^{2}},
$$


with notations as in Definition 2.4, and $k$ fixed and sufficiently large. Moreover, its first principal graph is given by the inclusion graph for $\bar{H}_{2|N| k} \subset \overline{\mathrm{Br}}_{2|N| k+m}$ for any sufficiently large $k$.

Proof. We first check conditions 1-4 of Section 1B with $A_{n}=\bar{H}_{n}$ and $B_{n}=\overline{\mathrm{Br}}_{n}(N)$ for $q=e^{\pi i / \ell}$ and $1<|N|<\ell$. Condition 1 is well-known and was checked, for instance, in [Wenzl 1988a]. Similarly, Condition 2 follows from the results in [Wenzl 2012], using the map $b \otimes g_{i} \in \overline{\mathrm{Br}}_{m} \otimes \bar{H}_{n} \mapsto b g_{m+i}$. Condition 3 means that the conditional expectation from $\overline{\mathrm{Br}}_{n+1}$ to $\overline{\mathrm{Br}}_{n}$ maps $\bar{H}_{n+1}$ onto $\bar{H}_{n}$. But as any element of $\bar{H}_{n+1}$ can be written as a linear combination of elements of the form $a g_{n} b$, with $a, b \in \bar{H}_{n}$, we have for any $c \in \overline{\mathrm{Br}}_{n}$ that

$$
\operatorname{tr}\left(a g_{n} b c\right)=\operatorname{tr}\left(g_{n}\right) \operatorname{tr}(a b c)=\operatorname{tr}\left(E_{\bar{H}_{n}}\left(a g_{n} b\right) c\right) .
$$

Hence the commuting square condition is satisfied for any four algebras of the type above. Finally, Condition 4 follows for $d=2 N$ and the projection $p=p_{\left[1^{N}\right]}^{\otimes 2 r}$ from Lemma 2.7.

The periodicity condition for $\bar{H}_{n}$ was shown in [Wenzl 1988a] by proving that $\bar{p}_{\left[1^{N}\right]} \bar{H}_{m+N} \bar{p}_{\left[1^{N}\right]} \cong \bar{H}_{m}$, for $N>0$. This induces an injective map

$$
\tilde{\Lambda}(N, \ell)_{m} \rightarrow \tilde{\Lambda}(N, \ell)_{m+N}
$$

by adding a column of $N$ boxes to the given Young diagram which has to become surjective for sufficiently large $m$ by definition of $\tilde{\Lambda}(N, \ell)$. The $2 N$ periodicity for the algebras $\overline{\mathrm{Br}}_{n}(N)$ follows similarly using Lemma 2.7; or, see [Wenzl 2012]. The reader should have no problem adjusting this proof to the case $N<0$ even, using Lemma 2.7.

\section{3. $S$-matrix}

We will need certain well-known identities, which can be found in [Kac 1990], except for one case, which is a variation of the other ones. Because of this, we review the material in more detail. This might also be useful to the nonexpert reader, as the identities needed here can be derived by completely elementary methods.

3A. Lattices. Let $M \subset L \subset \mathbb{R}^{k}$ be two lattices of full rank. This means that they are isomorphic to $\mathbb{Z}^{k}$ as abelian groups, and each of them spans $\mathbb{R}^{k}$ over $\mathbb{R}$. Moreover, we assume that we have an inner product on $\mathbb{R}^{k}$ such that $(\boldsymbol{x}, \boldsymbol{y}) \in \mathbb{Z}$ for all $\boldsymbol{x}, \boldsymbol{y} \in M$. We define the dual lattice $M^{*}$ to be the set of all $\boldsymbol{y} \in \mathbb{R}^{k}$ such that $(\boldsymbol{x}, \boldsymbol{y}) \in \mathbb{Z}$ for all $\boldsymbol{x} \in M$; the dual lattice $L^{*}$ is defined similarly. Obviously $M \subset L$ implies $L^{*} \subset M^{*}$. Finally, we also assume that $A=L / M$ is a finite abelian group. Then each $\gamma \in M^{*}$ defines a character of $A$ via the map $e^{\gamma}: \boldsymbol{x} \in L \mapsto e^{2 \pi i(\gamma, x)}$. In particular, one can 
identify the group dual of $A$ with $M^{*} / L^{*}$. Define the matrix

$$
\tilde{S}=\frac{1}{|L: M|^{1 / 2}}\left(e^{\gamma}(\boldsymbol{x})\right),
$$

where $\gamma$ and $x$ are representatives for the cosets $M^{*} / L^{*}$ and $L / M$. Then $\tilde{S}$ is the character matrix of $A$ up to a multiple and one easily concludes that it is unitary. More precisely, we can view it as a unitary operator between Hilbert spaces $V$ and $V^{*}$ with orthonormal bases labeled by the elements of $L / M$ and $M^{*} / L^{*}$ respectively.

3B. Weights of traces. We will primarily be interested in lattices related to root, coroot and weight lattices of orthogonal and symplectic groups. We define the lattices

$$
Q=\left\{\boldsymbol{x} \in \mathbb{Z}^{k}, 2 \mid \sum x_{i}\right\} \quad \text { and } P=\mathbb{Z}^{k} \cup\left(\varepsilon+\mathbb{Z}^{k}\right),
$$

where $\varepsilon$ is the element in $\mathbb{R}^{k}$ with all its coordinates equal to $1 / 2$. Observe that $P^{*}=Q$ with respect to the usual scalar product of $\mathbb{R}^{k}$. Moreover, one can identify coroot and weight lattices of $\mathfrak{s o}_{2 k}$ or $\mathfrak{s o}_{2 k+1}$ with $Q$ and $P$ respectively. In particular, we define for any $\gamma \in P$ the functional $e^{\gamma}: \mathbb{R}^{k} \rightarrow \mathbb{C}$ by $e^{\gamma}(\boldsymbol{x})=e^{2 \pi i(\gamma, \boldsymbol{x})}$. The Weyl group of type $B_{k}$ acts as usual via permutations and sign changes on the coordinates. Let $a_{W}=\sum_{w} \varepsilon(w) w$, where $\varepsilon(w)$ is the sign of the element $w$. Then the characters $\chi_{\lambda}$ for $\mathfrak{s o}_{2 k+1}$ and $\mathfrak{s p}_{2 k}$ are given by $\chi_{\lambda}=a_{w}\left(e^{\lambda+\rho}\right) / a_{w}\left(e^{\rho}\right)$, where $\rho=(k+1 / 2-i)$ for $\mathfrak{s o}_{2 k+1}$ and $\rho=(k+1-i)$ for $\mathfrak{s p}_{2 k}$, and $W$ is the Weyl group of type $B_{k}$.

We will also need the somewhat less familiar character formulas for the full orthogonal group $\mathrm{O}(N)$ : Recall that the irreducible representations of $\mathrm{O}(N)$ are labeled by Young diagrams $\lambda$ with at most $N$ boxes in the first two columns. $\mathrm{O}(N)$ modules labeled by Young diagrams $\lambda \neq \lambda^{\dagger}$ restrict to isomorphic $\mathrm{SO}(N)$-modules if and only if $\lambda_{1}^{\prime}=N-\left(\lambda^{\dagger}\right)_{1}^{\prime}$ and $\lambda_{i}^{\prime}=\left(\lambda^{\dagger}\right)_{i}^{\prime}$ for $i>1$. Hence if $g=\exp (\boldsymbol{x})$ is an element in $\mathrm{SO}(N)$, it suffices to consider the quantities $\chi_{\lambda}(g)=\chi_{\lambda}(\boldsymbol{x})$ for $\lambda$ with at most $k$ rows for $N=2 k$ or $N=2 k+1$. We can now express the weights of Theorem 2.8 in terms of these characters; in fact the formulas in Theorem 2.8 were derived from these characters; see [Koike 1997; Wenzl 2012].

Lemma 3.1. Let $d_{\lambda}, \tilde{d}_{\lambda}$ be as in Theorem 2.8 for $q=e^{\pi i / \ell}$. Moreover, we define for $|N|=2 k$ or $N=2 k+1$ the vector $\check{\rho} \in \mathbb{R}^{k}$ by $\check{\rho}=((|N|+1) / 2-i)_{i}$. By the discussion above, it suffices to evaluate $\chi^{\mathrm{O}(N)}(\check{\rho} / \ell)$ for Young diagrams $\lambda$ with $\lambda_{1}^{\prime} \leq N / 2$, which will be assumed in the following:

(a) If $N=2 k+1>0$, then $d_{\lambda}=\chi_{\lambda}^{\mathrm{O}(N)}(\check{\rho} / \ell)=\chi_{\lambda}^{\mathrm{SO}(N)}(\check{\rho} / \ell)$.

(b) If $N=2 k>0$ and $\lambda_{1}^{\prime} \leq k$, then $d_{\lambda}=m(\lambda) \operatorname{det}\left(\cos \left(l_{j} \check{\rho_{i}}\right) / \operatorname{det}\left(\cos (k-j) \check{\rho}_{i}\right)\right.$, where $l_{j}=(\lambda+\rho)_{j}=\lambda_{j}+k-j$ and where $m(\lambda)=2$ or 1 , depending on whether $\lambda$ has exactly $k$ rows or not. 
(c) If $N=-2 k$, then $d_{\lambda}=(-1)^{|\lambda|} \chi_{\lambda^{t}}^{\mathrm{Sp}(|N|)}(\check{\rho} / \ell)$ for the symplectic character labeled by the transposed diagram $\lambda^{T}$.

(d) We have $\tilde{d}_{\lambda}=\chi_{\lambda}^{\mathrm{SU}(N)}(\rho / \ell)$ for $N>0$ and $\tilde{d}_{\lambda}=(-1)^{|\lambda|} \chi_{\lambda^{T}}^{\mathrm{SU}(N)}(\rho / \ell)$ for $N<0$, where $\rho=((|N|+1) / 2-i) \in \mathbb{R}^{|N|}$.

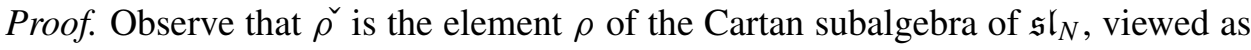
an element of the Cartan subalgebra of the Lie subalgebra $\mathfrak{s o}_{N}$ or $\mathfrak{s p}_{N}$, depending on the case. The proof now goes as the proof of Theorem 4.6 in [Wenzl 2012], which is essentially the one of [Koike 1997]. The fact that these arguments also work for the special quotients $\overline{\mathrm{Br}}_{n}$ follows from the proof of [Wenzl 2012, Theorem 5.5].

Remark 3.2. Let $\Delta_{+}$be the set of positive roots of a semisimple Lie algebra and $\left|\Delta_{+}\right|$be its cardinality. As usual, we can express the Weyl denominator in $\chi_{\lambda}(\check{\rho} / \ell)$ in product form as

$$
\Delta(\check{\rho} / \ell)=\prod_{\alpha>0}\left(e^{(\alpha, \check{\rho}) \pi i / \ell}-e^{-(\alpha, \check{\rho}) \pi i / \ell}\right)=(-i)^{\left|\Delta_{+}\right|} \prod_{\alpha>0} 2 \sin ((\alpha, \check{\rho}) \pi / \ell) .
$$

3C. Usual $S$-matrices. As usual, we pick as dominant chamber $C_{+}$the regions given by $x_{1}>x_{2}>\cdots>x_{k}>0$ for Lie types $B_{k}$ and $C_{k}$. We also choose the fundamental domains $D$ with respect to the translation actions of $M, M^{*}, L, L^{*}$ such that it has 0 in its center; here the lattices $M$ and $L$ will be certain multiples of the lattices $P, Q$ or $\mathbb{Z}^{k}$ to be specified later. Let $\bar{P}_{+}$be the intersection of $M^{*}$ with the fundamental alcove $D \cap C_{+}$.

Observe that we also obtain a representation of the Weyl group $W$ on the vector spaces $V$ and $V^{*}$. Then it is easy to check that $a_{W}\left(V^{*}\right)$ has an orthonormal basis $|W|^{-1 / 2} a_{w}\left(e^{\gamma}\right)$, with $\gamma \in \bar{P}_{+}$, and we can define a similar basis $a_{W}(\boldsymbol{x})$ for $a_{W}(V)$. Let $S$ be the matrix which describes the action of $\tilde{S}_{\mid a_{W}(V)}$ with respect to that basis. Then it is not hard to check (and we will do a slightly more complicated case below) that its coefficients are given by

$$
s_{\gamma, \boldsymbol{x}}=\frac{1}{|L: M|^{1 / 2}} \sum_{w} \varepsilon(w) e^{2 \pi i(w \cdot \gamma, \boldsymbol{x})} .
$$

If $L$ is the weight lattice of a simple Lie algebra, the entry $s_{\gamma, x}$ is the numerator of Weyl's character formula for the dominant weight $\lambda=\gamma-\rho$, up to the factor $|L: M|^{-1 / 2}$. As the columns of the unitary matrix $S$ have norm one, it follows that

$$
\sum_{\lambda} \chi_{\lambda}^{2}(\boldsymbol{x})=\frac{\mid L: M]}{\Delta^{2}(\boldsymbol{x})}
$$

where $\Delta$ is the Weyl denominator, and the summation goes over the dominant weights $\lambda$ such that $\lambda+\rho \in \bar{P}_{+}$. We are now in the position to prove some cases of the following proposition: 
Proposition 3.3. Let $\Lambda(N, \ell)_{e v}$ be the subset of $\Lambda(N, \ell)$ consisting of Young diagrams with an even number of boxes. Then we have

$$
\sum_{\lambda \in \Lambda(N, \ell)_{e v}} d_{\lambda}^{2}=\frac{\ell^{k}}{b(N)} \prod_{\alpha>0} \frac{1}{4 \sin ^{2}(\alpha, \check{\rho}) \pi / \ell},
$$

where $\tilde{\rho}=((|N|+1) / 2-i)$ and $\alpha>0$ runs through the positive roots of $\mathfrak{s o}_{N}$ for $N>0$ and of $\mathfrak{s p}_{|N|}$ for $N<0$ even, and $b(N)=2$ for $N=2 k>0$, and $b(N)=1$ otherwise.

Proof. Let us consider the case $N=2 k+1>0$, with $P$ and $Q$ as in (3-1). Let $L=\ell^{-1} \mathbb{Z}^{k}$ and let $M_{1}=Q$ and $M_{2}=\mathbb{Z}^{k}$. Then we have $M_{1}^{*}=P$, and $M_{2}^{*}=\mathbb{Z}^{k}$. Now observe that $M_{1}^{*}$ is the weight lattice of $\mathfrak{s o}_{N}$, and the elements $\gamma \in \bar{P}_{+}$are in 1-1 correspondence with the dominant weights $\lambda$ of $\mathfrak{s o}_{N}$ satisfying $\lambda_{1} \leq(\ell-N) / 2$, via the correspondence $\gamma=\lambda+\rho$. Moreover, $\left|L: M_{1}\right|=2 \ell^{k}$. Hence it follows from (3-4) that $\sum \chi_{\lambda}^{2}\left(\check{\rho}^{\check{ }}\right)=2 \ell^{k} / \Delta^{2}\left(\check{\rho}^{2}\right)$. Playing the same game for the lattice $M_{2}$, we now only get the sum over the characters $\chi_{\lambda}^{2}$ for which $\lambda+\rho$ is in $\mathbb{Z}^{k}$, which is only half as large as before. Hence also the sum over the characters $\chi_{\lambda}^{2}$ for which $\lambda \in \mathbb{Z}^{k}$ has to have the same value. This sum coincides with the right hand side of the statement for $N>0$ odd, by the restriction rules for $\mathrm{O}(N)$ to $\mathrm{SO}(N)$ (see Lemma 3.1 and its preceding discussion).

The symplectic case $N=-2 k<0$ goes similarly. Here we define $M \subset L=\ell^{-1} P$, and with $L^{*}=\ell Q \subset M^{*}=\mathbb{Z}^{k}$. Then it follows that $\sum d_{\lambda}^{2}=2 \ell^{k} / \Delta^{2}(\check{\rho} / \ell)$, where the summation goes over all diagrams $\lambda$ such that $\lambda^{T} \in \Lambda(N, \ell)$. Playing the same game for $M=P$ and $M^{*}=Q$, we get $\sum d_{\lambda}^{2}=\ell^{k} / \Delta(\tilde{\rho} / \ell)$, where now the summation goes over all even, or over all odd diagrams in $\Lambda(N, \ell)$, depending on whether the sum of coordinates of $\rho=(k+1-i)$ is odd or even. In each case, we obtain that $\sum_{e v} d_{\lambda}^{2}=\ell^{k} / \Delta(\check{\rho} / \ell)$. We have proved the proposition except for the case $N=2 k>0$, for which we need a little more preparation.

3D. Another S-matrix. We now consider a slight generalization of the above. Observe that we can define a second sign function $\tilde{\varepsilon}$ for $W=W\left(B_{k}\right)$ which coincides with the usual sign function on its normal subgroup $W\left(D_{k}\right)$, while we have $\tilde{\varepsilon}(w)=-\varepsilon(w)$ for $w \notin W\left(D_{k}\right)$. It is easy to see that also in this case we have $\tilde{\varepsilon}(v w)=\tilde{\varepsilon}(v) \tilde{\varepsilon}(w)$ for all $v, w \in W$. We define $\tilde{a}_{W}=\sum \tilde{\varepsilon}(w) w$, and also denote the corresponding operators on the various (quotient) lattices and on the vector spaces $V$ and $V^{*}$ by the same symbol. One observes that now we get an orthonormal basis for $\tilde{a}_{W}\left(V^{*}\right)$ of the form $\boldsymbol{b}_{\gamma}=|\operatorname{Stab}(\gamma)|^{-1 / 2}|W|^{-1 / 2} \tilde{a}_{W}\left(e^{\gamma}\right)$, labeled by the elements of $\bar{P}_{+}$which now consist of the $\gamma \in D$ such that $\gamma_{1}>\gamma_{2}>\cdots>\gamma_{k} \geq 0$. Observe that $|\operatorname{Stab}(\gamma)|$ is equal to 1 or 2 , depending on whether $\gamma_{k}>0$ or $\gamma_{k}=0$. One similarly defines a basis for $\tilde{a}_{W}(V)$. Let $\boldsymbol{x}$ be such that $\operatorname{Stab}(\boldsymbol{x})=1$, that is, 
$x_{k}>0$, and let $b_{x}=|W|^{-1 / 2} \tilde{a}_{W}(\boldsymbol{x})$. Then, writing $M^{*} / \ell L^{*}$ as a collection of $W$ orbits, we obtain

$$
\begin{aligned}
\tilde{\boldsymbol{S}} \boldsymbol{b}_{\boldsymbol{x}} & =|W|^{-1 / 2} \sum_{\lambda \in \bar{P}_{+}} \sum_{v, w \in W} \frac{1}{\left|\operatorname{Stab}_{W}(\gamma)\right|} \tilde{\varepsilon}(w) \tilde{s}_{v \cdot \gamma, w \cdot \boldsymbol{x}} v \cdot \gamma \\
& =\sum_{\lambda \in \bar{P}_{+}} \sum_{v}\left(\sum_{w} \tilde{\varepsilon}(w) \tilde{s}_{w \cdot \gamma, x} \frac{1}{\left|\operatorname{Stab}_{W}(\lambda)\right|}\right) \tilde{\varepsilon}(v) v \cdot \gamma,
\end{aligned}
$$

where we replaced $\tilde{\varepsilon}(w)$ by $\tilde{\varepsilon}(v) \tilde{\varepsilon}\left(w^{-1} v\right), \tilde{s}_{v, \lambda, w . x}$ by $\tilde{s}_{w^{-1} v \cdot \gamma, x}$ and finally also substituted $w^{-1} v$ by $w$. We see from this that the coefficient of $v \cdot \gamma$ is equal to 0 if $\gamma$ has a nontrivial stabilizer except in the case when $\gamma_{k}=0$. Hence it follows that $\tilde{S}$ maps $a_{W}(V)$ into $a_{W}\left(V^{*}\right)$. Taking bases $\left(\tilde{a}_{W}(\gamma)\right)_{\gamma \in P_{+}}$and $\left(\tilde{a}_{W}(\boldsymbol{x})\right)$, we see that $\tilde{S}_{\mid a_{W}(V)}$ can be described by the matrix $S=\left(s_{\gamma, x}\right)$ whose coefficients are given for $\boldsymbol{x}$ with trivial stabilizer by

$$
s_{\gamma, \boldsymbol{x}}=|\operatorname{Stab}(\gamma)|^{-1 / 2}|L: M|^{-1 / 2} \sum_{w} \varepsilon(w) e^{2 \pi i(w \cdot \gamma, \boldsymbol{x})} .
$$

3E. Squares of characters. Using the discussion from before and the formulas of Lemma 3.1 it is not hard to see that for $N$ even and $\lambda_{1}^{\prime} \leq N / 2$ we can write

$$
\chi_{\lambda}^{\mathrm{O}(N)}=m(\lambda) \tilde{a}_{W}\left(e^{\lambda+\rho}\right) / \tilde{a}_{W}\left(e^{\rho}\right),
$$

where $m(\lambda)=2$ or 1 depending on whether $\lambda$ has exactly $k$ rows or not. In particular, applying this to the trivial representation, we obtain $2 \Delta(\rho)=\tilde{a}_{W}\left(e^{\rho}\right)$.

Let $P$ and $Q$ be as in (3-1), and set $L=\ell^{-1} P$ and $M=\mathbb{Z}^{k}$. Then

$$
L^{*}=\ell Q \subset M^{*}=\mathbb{Z}^{k},
$$

and it is easy to see that all of these lattices are $W=W\left(B_{k}\right)$-invariant. Moreover, let $\check{\rho} / \ell=(k+1 / 2-i) / \ell \in \ell^{-1} P=M^{*}$. Then it follows for $N=2 k$ and $\ell$ even that

$$
\sum_{\lambda \in \Lambda(N, \ell)} \chi_{\lambda}^{2}(\check{\rho} \ell)=\frac{1}{\Delta^{2}(\check{\rho} \ell)} \sum_{\substack{\lambda_{k+1}=0 \\ \lambda_{1} \leq(\ell-N) / 2}}\left(\tilde{a}_{W}\left(e^{\lambda+\rho}\right)(\check{\rho}) \ell\right)^{2}=\frac{\mid L: M]}{2 \Delta^{2}(\check{\rho})} \sum_{\lambda} s_{\lambda, \check{\rho} / \ell}^{2} .
$$

Now observe that the matrix $S$ is unitary and that $[L: M]=2 \ell^{k}$. Moreover, by Proposition 1.1 and Theorem 2.11, the square sum over odd diagrams must be equal to the square sum over even diagrams. Hence we obtain for $N>0$ even, and $\ell$ even that

$$
\sum_{\lambda \in \Lambda(N, \ell)_{e v}} d_{\lambda}^{2}=\frac{\ell^{k}}{2 \Delta^{2}(\check{\rho})}
$$


where $\Lambda(N, \ell)_{e v}$ denotes the set of diagrams in $\Lambda(N, \ell)$ with an even number of boxes. This finishes the last case of the proof of Proposition 3.3

3F. Calculation of index. As usual, identify the Cartan algebra of $\mathfrak{s l}_{N}$ with the diagonal $N \times N$ matrices with zero trace. The embedding of the Cartan algebras of an orthogonal or symplectic subalgebra is given via diagonal matrices for which the $(N+1-i)$-th entry is the negative of the $i$-th entry, for $1 \leq i \leq N / 2$. Hence, if $\epsilon_{i}$ is the $\mathfrak{s l}_{N}$ weight given by the projection onto the $i$-th diagonal entry, we have $\left(\epsilon_{N+1-i}\right)_{\mid \mathfrak{s o}_{N}}=\left(-\epsilon_{i}\right)_{\mid \mathfrak{s o}_{N}}$, with a similar identity also holding for symplectic subalgebras. Using our description of coroot and weight lattices of orthogonal and symplectic Lie algebras as sublattices of $\mathbb{R}^{k}$, and defining $\phi_{i}$ to be the projection onto the $i$-coordinate, we see that $\left(\epsilon_{N+1-i}\right)_{\mid \mathfrak{s o}_{N}}=-\phi_{i}=\left(-\epsilon_{i}\right)_{\mid \mathfrak{s o}_{N}}$. This allows us to describe the decomposition of $\mathfrak{s l}_{N}$ as both an $\mathfrak{s o}_{N}$ - and $\mathfrak{s p}_{N}$-module:

$$
\mathfrak{s l}_{N}=\mathfrak{s o}_{N} \oplus \mathfrak{p} \quad \text { and } \quad \mathfrak{s l}_{N}=\mathfrak{s p}_{N} \oplus \mathfrak{p},
$$

where $\mathfrak{p}$ denotes, respectively, the nontrivial irreducible submodule in the symmetrization of the vector representation of $\mathfrak{s o}_{N}$, and the nontrivial irreducible submodule in the antisymmetrization of the vector representation of $\mathfrak{s p}_{N}$. The nonzero weights $\omega>0$ of $\mathfrak{p}$ coming from positive roots of $\mathfrak{s l}_{N}$ and the multiplicity $n(\mathfrak{p})$ of the weight 0 in $\mathfrak{p}$ are given by

(a) $2 \phi_{i}, \phi_{i}$ and $\phi_{i} \pm \phi_{j}$ for $1 \leq i<j \leq k$ with $n(\mathfrak{p})=k$ for $\mathfrak{s o}_{N}$ with $N=2 k+1$ odd,

(b) $2 \phi_{i}$ and $\phi_{i} \pm \phi_{j}$ for $1 \leq i<j \leq k$ with $n(\mathfrak{p})=k-1$ for $\mathfrak{s o}_{N}$ with $N=2 k$ even,

(c) $\phi_{i} \pm \phi_{j}$ for $1 \leq i<j \leq k$ with $n(\mathfrak{p})=k-1$ for $\mathfrak{s p}_{|N|}$ with $N=-2 k<0$ even.

Theorem 3.4. The index of the subfactor $\mathcal{N} \subset M$ obtained from the inclusions of algebras $\bar{H}_{n}(q) \subset \overline{\mathrm{Br}}_{n}\left(q^{N}, q\right)$ is given by

$$
[\mu: \mathcal{N}]=b(N) \ell^{n(\mathfrak{p})} \prod_{\omega>0} \frac{1}{4 \sin ^{2}\left(\omega, \rho^{\check{ }}\right) \pi / \ell},
$$

where the product goes over the weights $\omega>0$ of $\mathfrak{p}$ coming from positive roots of $\mathfrak{s l}_{N}$, as listed above, $n(\mathfrak{p})$ is the multiplicity of the zero weight in $\mathfrak{p}$, and $b(N)$ and $\check{\rho}$ are as in Proposition 3.3.

Corollary 3.5. If $q=e^{\pi i / \ell} \rightarrow 1$, the index $[\mathcal{M}: \mathcal{N}]$ goes to $\infty$ with asymptotics $\ell^{\operatorname{dim} \mathfrak{p}}$. Proof. We use Theorem 2.11, where the denominator has been calculated in Proposition 3.3. The numerator follows from a standard argument for $S$-matrices for Lie type $A$; see [Kac 1990], versions of which have also been used in this section. For an elementary calculation, see [Erlijman 1998].

Remark 3.6. It is straightforward to adapt our index formula to subfactors related to other fixed points $H=G^{\alpha}$ of an order two automorphism $\alpha$ of a compact Lie 
group $G$, up to some integer (or perhaps rational) constant $b(H, G)$. Again, $\mathfrak{p}$ would be the -1 eigenspace of the induced action of $\alpha$ on the Lie algebra $\mathfrak{g}$, and the same $S$-matrix techniques applied in this section would go through. For example, our formulas for $N=3$ and $\ell$ odd coincide with the ones at the end of [Xu 2009] for even level of SU(3), up to a factor 3 (and missing squares, a misprint according to the author). This is to be expected as in our case only those diagrams appear in the principal graph (see next section) which also label representations of the projective group PSU(3).

3G. Restriction rules and principal graph. It follows from Theorem 1.4 that the principal graph of $\mathcal{N} \subset \mathcal{M}$ is given by the inclusion matrix for $\bar{H}_{2 k} \subset \overline{\mathrm{Br}}_{2 k}$ for $k$ sufficiently large. This still leaves the question of how to explicitly calculate these graphs. Observe that in the classical case $q=1$ these would be given by the restriction rules from the unitary group $\mathrm{U}(N)$ to $\mathrm{O}(N)$, for $N>0$. Formulas for these restriction coefficients are well-known; see for instance [Weyl 1997, Theorems 7.8F and 7.9C] and Littlewood's formula (see [Koike and Terada 1987, Section 1.5], and the whole paper for additional results). Another approach closely related to the setting of fusion categories can also be found in [Wenzl 2011].

Let $b_{\mu}^{\lambda}(N)$ be the multiplicity of the simple $\mathrm{O}(N)$-module $V_{\mu}$ in the $\mathrm{U}(N)$ module $F_{\lambda}$, for $N>0$, where $\lambda, \mu$ are Young diagrams. It is well-known that for fixed Young diagrams $\lambda$ and $\mu$, the number $b_{\mu}^{\lambda}(N)$ will become a constant $b_{\mu}^{\lambda}$ for $N$ large enough. Fix now also $\ell>|N|$. We define similar coefficients in our setting as follows: Recall that the simple components of $\bar{H}_{n}$ are labeled by the diagrams in $\tilde{\Lambda}(N, \ell)_{n}$ and the ones of $\overline{\mathrm{Br}}_{n}$ by the diagrams in $\Lambda(N, \ell)$. We then define for $\lambda \in \tilde{\Lambda}(N, \ell)$ and $\mu \in \Lambda(N, \ell)$ the number $b_{\mu}^{\lambda}(N, \ell)$ to be the multiplicity of a simple $\bar{H}_{n, \lambda}$ module in a simple $\overline{\mathrm{Br}}_{n, \mu}$ module.

In the following lemma the symbol $\chi_{\mu}$ will also be used for the $\mathrm{O}(N)$ character corresponding to the simple representation labeled by the Young diagram $\mu$. Moreover, we also denote by $\overline{\mathrm{Br}}_{\infty}$ the inductive limit of the finite dimensional algebras $\overline{\mathrm{Br}}_{n}$ under their standard inclusions, for fixed $N$ and $\ell$.

Lemma 3.7. (a) Each $g \in O(N)$ for which $\chi_{\mu}(g)=0$ for all boundary diagrams $\mu$ of $\Lambda(N, \ell)$ defines a trace on $\overline{\operatorname{Br}}_{\infty}$ determined by $\operatorname{tr}\left(p_{\mu}\right)=\chi_{\mu}(g) / \chi_{[1]}(g)^{n}$, where $p_{\mu}$ is a minimal projection of $\overline{\mathrm{Br}}_{n, \mu}$.

(b) For given $\lambda \in \tilde{\Lambda}(N, \ell)_{n}$ the coefficients $b_{\mu}^{\lambda}(N, \ell)$ are uniquely determined by the equations

$$
\chi_{\lambda}^{\mathrm{U}(N)}(g)=\sum_{\mu} b_{\mu}^{\lambda}(N, \ell) \chi_{\mu}(g)
$$

for all $g$ as in $(a)$, where the summation goes over all diagrams $\mu$ in $\Lambda(N, \ell)$ with $n, n-2, \ldots$ boxes. 
Proof. The formula in statement (a) determines a trace on $\overline{\mathrm{Br}}_{n}$ for each $n$. To show that these formulas are compatible with the standard embeddings we observe that a minimal idempotent $p_{\mu} \in \overline{\mathrm{Br}}_{n, \mu}$ is the sum of minimal idempotents $e_{\lambda} \in \overline{\mathrm{Br}}_{n+1, \lambda}$ where $\lambda$ runs through all diagrams in $\Lambda(N, \ell)$ obtained by adding or removing a box to/from $\lambda$; see (2-2) and the remarks below that theorem. Evaluating the traces of these idempotents and multiplying everything by $\chi_{\lambda}(g)^{n+1}$, equality of the traces is equivalent to

$$
\chi_{\mu}(g) \chi_{[1]}(g)=\sum_{\lambda} \chi_{\lambda}(g)
$$

By the usual tensor product rule for orthogonal groups, the left hand side would be equal to the sum of characters corresponding to all diagrams $\lambda$ which differ from $\mu$ by only one box. It is easy to check that this differs from the sum above only by boundary diagrams, for which the characters at $g$ are equal to 0 . This shows (a).

For (b), we first show that

$$
\operatorname{tr}\left(p_{\lambda}\right)=\frac{\chi_{\lambda}^{\mathrm{U}(N)}(g)}{\chi_{[1]}^{\mathrm{U}(N)}(g)^{n}}
$$

for $p_{\lambda} \in \bar{H}_{n, \lambda}$ a minimal idempotent and tr a trace as in (a). As the weight vector for $\overline{\mathrm{Br}}_{n+2 N}$ is a multiple of that of $\overline{\mathrm{Br}}_{n}$, for $n$ large enough, the same must also hold for the weight vectors of $\bar{H}_{n}$ and $\bar{H}_{n+2 N}$, by periodicity of the inclusions. Hence, these weight vectors must be eigenvectors of the inclusion matrix for $\bar{H}_{n} \subset \bar{H}_{n+2 N}$. As this inclusion matrix is just a block of the $2 N$-th power of the fusion matrix of the vector representation for the corresponding type $A$ fusion category, its entries must be given by $\mathrm{U}(N)$ characters of a suitable group element. To identify these elements, it suffices to observe that the antisymmetrizations of the vector representation, labeled by the Young diagrams $\lambda=\left[1^{j}\right], 1 \leq j \leq N$, remain irreducible as $\mathrm{O}(N)$ modules. This means the corresponding Hecke algebra idempotent remains a minimal idempotent also in $\overline{\operatorname{Br}}_{j}$. Hence $\operatorname{tr}\left(p_{\lambda}\right)=\chi_{\lambda}^{\mathrm{U}(N)}(g)$ for $\lambda=\left[1^{j}\right]$ and $1 \leq j \leq N$. But as the antisymmetrizations generate the representation ring of $\mathrm{U}(N)$, and also of the corresponding fusion ring, the claim follows for general $\lambda$. For more details, see [Goodman and Wenzl 1990].

Recall that the coefficient $b_{\mu}^{\lambda}(N, \ell)$ can be defined as the rank of $p_{\lambda}$ in an irreducible $\overline{\mathrm{Br}}_{n, \mu}$ representation. So obviously the formula in the statement holds for any $g$ as in (a). Examples for such $g$ come from $\exp (\boldsymbol{x})$ with $\boldsymbol{x} \in M^{*}=\ell^{-1} Q$ for which the character is given by the expression $\chi_{\lambda}(\boldsymbol{x})$ as in Section $2 \mathrm{E}$. As the columns of the orthogonal $S$-matrix are linearly independent, this would identify $\mathrm{SO}(N)$ representations. If $N$ is odd, the two $\mathrm{O}(N)$ representations which reduce to the same $\mathrm{SO}(N)$ representation are labeled by Young diagrams with opposite 
parities. Hence only one of them can occur in the decomposition of a given $\mathrm{U}(N)$ representation. A similar argument also works in the symplectic case.

For $N$ even, we can have two diagrams $\lambda$ and $\lambda^{\dagger}$ with the same $\operatorname{SO}(N)$ character, where one of them, say $\lambda$, has less than $k$ rows. They can be distinguished by elements $g \in O(N) \backslash \mathrm{SO}(N)$ for which $\chi_{\lambda^{\dagger}}(g)=-\chi_{\lambda}(g)$. It is well-known that such elements $g$ must have eigenvalues \pm 1 , and $\chi_{\lambda}(g)$ is given by the character formula for $\mathrm{Sp}(2 k-2)$ in the remaining $2 k-2$ eigenvalues; see [Weyl 1997]. It follows from the invertibility of the $S$-matrix for $\operatorname{Sp}(2 k-2)$ at level $\ell / 2-k$ (see [Kac 1990]) that we can identify those diagrams $\lambda$ by evaluating $\chi_{\lambda}^{\operatorname{Sp}(2 k-2)}(\boldsymbol{x} / \ell)$ for $\boldsymbol{x} \in \mathbb{Z}^{k-1}$ with $\ell / 2>x_{1}>x_{2}>\cdots>x_{k-1}>0$, and that those elements satisfy the boundary condition $\chi_{\lambda}^{\mathrm{Sp}(2 k-2)}(\boldsymbol{x})=0$ for any boundary diagram $\lambda$.

The lemma above is illustrated in the following section for a number of explicit examples. We can also give a closed formula for the restriction coefficients, using a well-known quotient map for fusion rings (even though in our case, the quotient ring does not correspond to a tensor category as far as we know). In the context of fusion rings, this is known as the Kac-Walton formula; for type $A$ see also [Goodman and Wenzl 1990]. In our case, we need to use a slightly different affine reflection group $\mathcal{W}$. In the orthogonal case $N=2 k$ and $N=2 k+1$ it is given by the semidirect product of $\ell \mathbb{Z}^{k}$ with the Weyl group of type $B_{k}$. In the symplectic case, it is given by the semidirect product of $\ell Q$ with the Weyl group of type $B_{k}$. As usual, we define the dot action of $\mathcal{W}$ on $\mathbb{R}^{k}$ by $w \cdot \boldsymbol{x}=w(\boldsymbol{x}+\rho)-\rho$, where $\rho$ is half the sum of the positive roots of the corresponding Lie algebra, with the roots embedded into $\mathbb{R}^{k}$ as described above, and $\varepsilon$ is the usual sign function for reflection groups. This can be extended to an action on the labeling set of $\mathrm{O}(N)$ representations by identifying a Young diagrams with at most $k$ rows with the corresponding vector in $\mathbb{Z}^{k}$, and by using the restriction rule from $\mathrm{O}(N)$ to $\mathrm{SO}(N)$ in the other cases. See also [Wenzl 2011, Lemma 1.7] for more details.

Theorem 3.8. With notations as above, the restriction multiplicity $b_{\mu}^{\lambda}(N, \ell)$ for $N=2 k+1>0$ and $N=-2 k$ is given by

$$
b_{\mu}^{\lambda}(N, \ell)=\sum_{w \in \mathscr{W}} \varepsilon(w) b_{w \cdot \mu}^{\lambda}(N) .
$$

If $N=2 k>0$, we have to replace $\varepsilon$ by $\tilde{\varepsilon}$ (see Section $3 D$ ) in the formula above.

Proof. Looking at the character formulas, we see that an action of an element $w$ of the finite reflection group on $\lambda$ just changes the character by the sign of $w$. Moreover, by definition of the elements $\boldsymbol{x}$ we have that $\chi_{\lambda}(\boldsymbol{x})=\chi_{\lambda+\mu}(\boldsymbol{x})$ for any $\mu \in M$. It follows that $\chi_{w . \lambda}(\boldsymbol{x})=\varepsilon(w) \chi_{\lambda}(\boldsymbol{x})$ for all $\boldsymbol{x} \in M^{*}$ and $w \in \mathcal{W}$. Hence summing 
over the $\mathcal{W}$-orbits, we obtain for any $\boldsymbol{x} \in M^{*}, \lambda \in \tilde{\Lambda}(N, \ell)$ and $\mu \in \Lambda(N, \ell)$ that

$$
\chi_{\lambda}^{\mathrm{U}(N)}(\boldsymbol{x})=\sum_{\gamma} b_{\gamma}^{\lambda}(N) \chi_{\gamma}=\sum_{\mu}\left(\sum_{w} b_{w . \mu}^{\lambda}(N)\right) \chi_{\mu} .
$$

The claim now follows from this and Lemma 3.7.

\section{Examples and other approaches}

4A. The case $N=2$. This corresponds to the Goodman-de la Harpe-Jones subfactors for type $D_{\ell / 2+1}$, where $\ell>2$ has to be even. It follows from our theorem that the even vertices of the principal graph are labeled by the Young diagrams $\lambda$ with an even number $n$ of boxes, at most two rows and with $\lambda_{1}-\lambda_{2} \leq \ell-2$; there are $(\ell-2) / 2$ such diagrams. Their dimensions are given by $\tilde{d}_{k}=[2 k+1]$, for $0 \leq k<(\ell-2) / 2$.

Moreover, one checks that $\Lambda(2, \ell)$ consists of Young diagrams $[j]$ such that $0 \leq j \leq(\ell / 2)-1$ and of $\left[1^{2}\right]$, one column with 2 boxes, with dimensions $d_{[j]}=2 \cos j \pi / \ell$ for $j>0$ and dimension equal to 1 for the remaining cases (that is, for $\varnothing$ and for $\left[1^{2}\right]$ ). The restriction rule (that is, principal graph) follows from writing the dimensions as

$$
\tilde{d}_{k}=2 \cos \tilde{k} \pi / \ell+2 \cos (\tilde{k}-2) \pi / \ell+\cdots+1,
$$

where $\tilde{k}=\min \{k,(\ell / 2)-k\}$. Indeed, this determines the graph completely except for whether to pick the diagram $\varnothing$ or $\left[1^{2}\right]$ for the object with dimension 1 . It follows from the restriction rule $\mathrm{O}(2) \subset U(2)$ that we take $\varnothing$ for $j$ even, and [1 $\left.{ }^{2}\right]$ for $j$ odd. To calculate the index one can check by elementary means that $\sum_{\lambda \text { even }} d_{\lambda}^{2}=\ell / 2$. Moreover, it is well-known that the sum $\sum_{\lambda \text { even }} \tilde{d}_{\lambda}^{2}$ over even partitions for $\mathfrak{s l}_{2}$ is equal to $\ell /\left(4 \sin ^{2} \pi / \ell\right)$. Hence we obtain as index $[\mathcal{M}: \mathcal{N}]=1 /\left(2 \sin ^{2} \pi / \ell\right)$.

4B. The case $N=3$. It is also fairly elementary to work out this case in detail. A detailed discussion of $\mathrm{SU}(3)$ fusion modular categories has already been given in [Ocneanu 2002] (without proofs) and in [Evans and Pugh 2011] and references therein. These include our examples here. Recall that by Weyl's dimension formula we have

$$
\tilde{d}_{\lambda}=\frac{\left[\lambda_{1}-\lambda_{2}+1\right]\left[\lambda_{2}-\lambda_{3}+1\right]\left[\lambda_{1}-\lambda_{3}+2\right]}{[1]^{2}[2]} .
$$

Now observe that the product of two $q$-numbers is given by the tensor product rules for $\mathfrak{s l}_{2}$, that is, we have for $n \geq m$ that

$$
[n][m]=[n+m-1]+[n+m-3]+\cdots+[n-m+1] .
$$




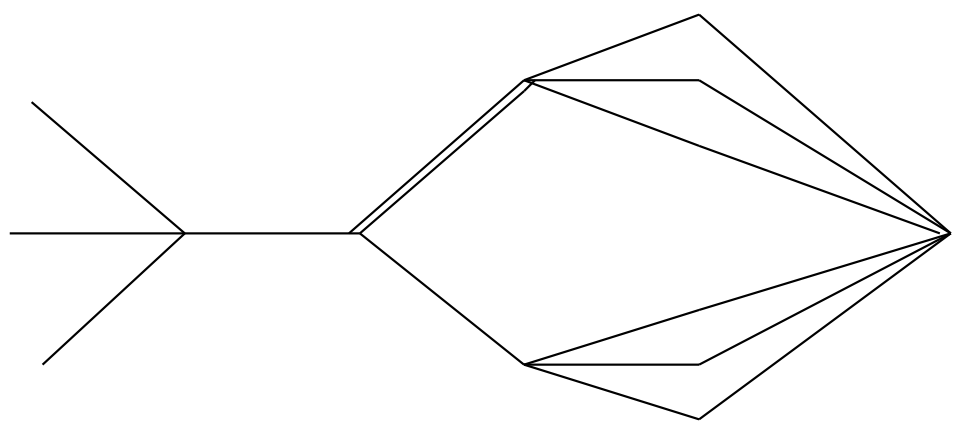

Figure 1. The principal graph of $\mathrm{SO}(3)$ for $\ell=9$.

As an example, we have

$$
\tilde{d}_{[4]}=\frac{[6][5]}{[2]}=\frac{[10]+[8]+[6]+[4]+[2]}{[2]}=[9]+[5]+[1],
$$

that is, the fourth antisymmetrization of the vector representation of $U(3)$ decomposes as a direct sum of the one-, five- and nine-dimensional representation of $\mathrm{SO}(3)$. One similarly can show the well-known result that the adjoint representation of SU(3), labeled by the Young diagram $[2,1]$ decomposes into the direct sum of the three- and the five-dimensional representation of $\mathrm{SO}(3)$, that is, $\mathfrak{p}$ is the five dimensional representation of $\mathrm{SO}(3)$. Hence we get from Theorem 3.4 that the index is equal to

$$
[M: \mathcal{N}]=\frac{\ell}{4^{2} \sin ^{2}(2 \pi / \ell) \sin ^{2}(\pi / \ell)} .
$$

We note that here as well as in the other examples, the dimensions (that is, entries of the Perron-Frobenius vectors) are given by $\left|\tilde{d}_{\lambda}\right|$ for even vertices, and by $\sqrt{[\mathcal{M : \mathcal { N }}]}\left|d_{\mu}\right|$ for odd vertices, with $\tilde{d}_{\lambda}$ and $d_{\mu}$ as in Lemma 3.1. To consider explicit examples, the first nontrivial case for $N=3$ occurs for $\ell=7$. We leave it to the reader to check that in this case the first principal graph is given by the Dynkin graph $D_{8}$. A more interesting graph is obtained for $\ell=9$; see Figure 1 . Here we have the three invertible objects of the $\mathrm{SU}(3)_{6}$ fusion category, including the trivial object (often denoted as $*$ ) on the left; they generate a group isomorphic to $\mathbb{Z} / 3$. The vertices with the double edge are labeled by the object corresponding to the 5-dimensional representation of $\mathrm{SO}(3)$ and the diagram $[4,2]$ for $\mathrm{SU}(3)_{6}$. This is the only fixed point under the $\mathbb{Z} / 3$ action given by the invertible objects (or, in physics language, the currents). It would be interesting to see whether one can carry out an orbifold construction in this context related to the one in [Evans and Kawahigashi 1994]. 
4C. The case $N=4$. The combinatorics of these subfactors has already appeared in [Ocneanu 2002; Evans and Gannon 2010] and also in the mathematical physics literature (see below), but the author is not aware of a rigorous general construction of the subfactors in the literature (but see the remarks below about the work in [Wassermann 2010; Xu 2009]). As we shall see, somewhat surprisingly, the corresponding construction for $\mathrm{SO}(4)$ does not seem to work. We do the case with $\ell=8$ in explicit detail. It is not hard to check that we already get the periodic inclusion matrix for $n=12$. As we consider an analog of the restriction to $\mathrm{O}(4)$ for which the determinant can be \pm 1 , we should, strictly speaking, consider a fusion category for $\mathrm{SU}(4) \times\{ \pm 1\}$. We shall actually use the Young diagram notation for representations of $\mathrm{U}(4)$. For $n=12$ we have the invertible objects labeled by [ $3^{4}$ ], $\left[4^{3}\right],\left[5^{2} 1^{2}\right]$ and $\left[62^{3}\right]$ (that is, the last diagram, for instance, has six boxes in the first and two boxes each in the second, third and fourth rows). They generate a subgroup isomorphic to $\mathbb{Z} / 4$. It follows from the $\mathrm{O}(4)$ restriction rules that $\left[3^{4}\right]$ and $\left[5^{2} 1^{2}\right]$ contain the determinant representation, and $\left[4^{3}\right]$ and $\left[62^{3}\right]$ contain the trivial representation as one-dimensional $\mathrm{O}(4)$ subrepresentations. This allows us to calculate the restrictions for representations of each $\mathbb{Z} / 4$ orbit simultaneously. As usually for at least one element of each orbit the ordinary restriction rules still hold, it makes the general calculations easier. The principal graph can be seen in Figure 2. As in the $N=3$ example, the one-dimensional currents, including the trivial object $*$ appear as the left- and right-most vertices in the graph. The lowest vertex corresponds to the $\mathrm{O}(4)$-object [2] which is connected to the objects in the $\mathbb{Z} / 4$-orbit $\left\{\left[2,1^{2}\right],[3,1],[4,3,1],[3,3,2]\right\}$. We also note that we get the same graph for the $\mathrm{Sp}(4)$ case $N=-4$ for $\ell=8$. However, for other roots of unity, already the indices of the subfactors differ, being given by

$$
\mathrm{O}(4): \frac{2 \ell}{4 \sin ^{2}(3 \pi / \ell) 4 \sin ^{2}(2 \pi / \ell) 16 \sin ^{4}(\pi / \ell)} \quad \mathrm{Sp}(4): \frac{\ell}{4 \sin ^{2}(2 \pi / \ell) 4 \sin ^{2}(\pi / \ell)} .
$$

It was originally thought that we should also be able to get fusion category analogs for the restriction from $\mathrm{SU}(N)$ to $\mathrm{SO}(N)$ for $N$ even. It is easy to check that this is not possible for $\mathrm{O}(2)$. Some initial checks also seem to suggest a similar phenomenon for higher ranks. For example, using the same element $\check{\rho}$ in the $\mathrm{SO}(N)$ character formula would give dimension functions which are not invariant under the $D_{N}$ diagram automorphism.

4D. Related results. We discuss several results related to our findings. Our original motivation was to construct subfactors related to twisted loop groups. It was shown in R. Verrill's PhD thesis [2001] that it is not possible to construct a fusion tensor product for representations of twisted loop groups. However, it seemed reasonable to expect that representations of twisted loop groups could become a module category over representations of their untwisted counterparts. Many results, in 


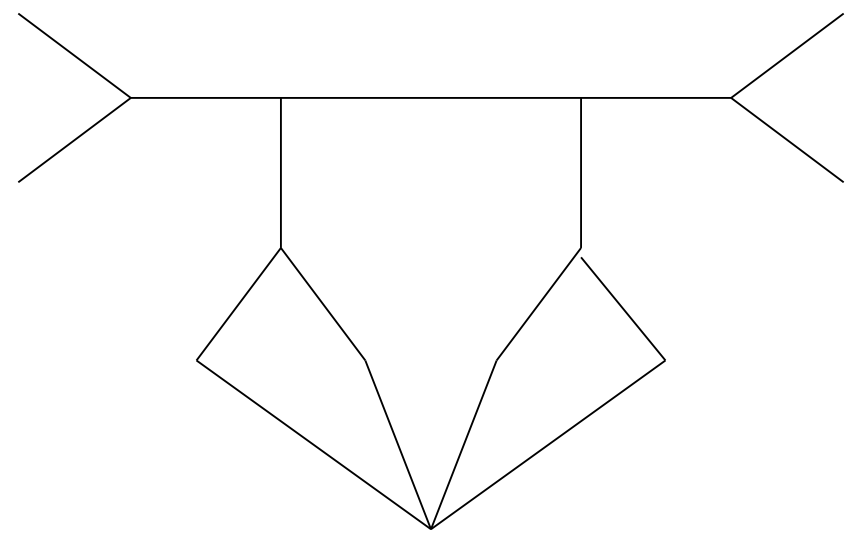

Figure 2. The principal graph of $\mathrm{O}(4)$ and $\mathrm{Sp}(4)$ for $\ell=8$.

particular about the combinatorics of such categories, can be found in the context of boundary conformal field theory in papers by Evans, Gaberdiel, Gannon, Fuchs, Pugh, Schweigert, Di Francesco, Petkova, Zuber and others; see for instance [Evans and Gannon 2010; Gaberdiel and Gannon 2002; Fuchs and Schweigert 2000; Petkova and Zuber 2002] and the papers cited therein. Understanding results in these papers in mathematical terms was one of the motivations for this author. Similarly, mathematical results in [Ocneanu 2002; Evans and Pugh 2011] for the cases $N=3$ and $N=4$ (see the introduction) were influenced by these papers, in particular by work of Zuber and his coauthors.

In the mathematics literature, one can find closely related results in [Xu 2009; Wassermann 2010]. Here the authors construct module categories via a completely different approach in the context of type $\mathrm{III}_{1}$ factors, using loop groups. For instance, the formulas at the end of [Xu 2009] for the special case $N=3$ differ only by a factor 3 (which can be explained; see Remark 3.6), by our formulas for $N=3$ for even level (together with Corollary 1.5), modulo misprints. Similar formulas for the symplectic case as well as restriction coefficients also appear at the end of [Wassermann 2010]. We cannot get results corresponding to the odd level cases in [Xu 2009]. The combinatorics there suggests that this would require considering an embedding of $\operatorname{Sp}(N-1)$ into $\mathrm{SU}(N)$ under which the vector representation would not remain irreducible. In contrast, we can also construct module categories for $\ell-N$ odd, which would correspond to odd level; however, these categories are not unitarizable (which follows from Lemma 2.9) and they have different fusion rules. However, we do get fairly general formulas for the index and principal graphs of this type of subfactors in the unitary case, which was one of the problems posed in [Xu 2009]. These formulas were known to this author as well as to Antony 
Wassermann at least back in 2008 when they had discussions about their respective works in Oberwolfach and at the Schrödinger Institute.

We close this section by mentioning that while our results for $N>0$ odd and $N<0$ even are in many ways parallel to results obtained via other approaches in connection with twisted loop groups, there does not seem to be an obvious analog for our results for $N>0$ even. For instance, the combinatorial results in [Gaberdiel and Gannon 2002] for that case seem to be different to ours.

4E. Conclusions and further explorations. We have constructed module categories of fusion categories of type $A$ via deformations of centralizer algebras of certain subgroups of unitary groups. We have also classified when they are unitarizable, we have constructed the corresponding subfactors, and we have explicitly calculated their indices and first principal graphs. These deformations are compatible with the Drinfeld-Jimbo deformation of the unitary group but not with the Drinfeld-Jimbo deformation of the subgroup. Most of the deformation was already done in [Wenzl 2012] via elementary methods. In principle, at least, it should be possible to use this elementary approach also for other inclusions. However, this might become increasingly tedious.

As we have seen already in Section 2B, it should be possible to get a somewhat more conceptual approach using different deformations of the subgroup; see [Noumi 1996; Molev 2003; Letzter 1997; Letzter 2002; Iorgov and Klimyk 2005] and references therein. In particular in the work of Letzter, such deformations via coideal algebras have been defined for a large class of embeddings of a semisimple Lie algebra into another one. At this point, it does not seem obvious how to define $C^{*}$-structures in this setting, and additional complications arise as these coideal algebras are not expected to be semisimple at roots of unity. Nevertheless, the results in this and other papers such as [Xu 2009; Wassermann 2010] would seem to suggest that similar constructions might be possible also in a more general setting.

\section{Acknowledgments}

It is a pleasure to thank Antony Wassermann, David Jordan, Viktor Ostrik, Feng $\mathrm{Xu}$ and David Evans for useful references, and Fred Goodman also for technical advice.

\section{References}

[Bisch 1997] D. Bisch, "Bimodules, higher relative commutants and the fusion algebra associated to a subfactor", pp. 13-63 in Operator algebras and their applications (Waterloo, ON, 1994/1995)), edited by P. A. Fillmore and J. A. Mingo, Fields Inst. Commun. 13, Amer. Math. Soc., Providence, RI, 1997. MR 97i:46109 Zbl 0894.46046 
[Böckenhauer et al. 1999] J. Böckenhauer, D. E. Evans, and Y. Kawahigashi, "On $\alpha$-induction, chiral generators and modular invariants for subfactors", Comm. Math. Phys. 208:2 (1999), 429-487. MR 2001c:81180 Zbl 0948.46048

[Erlijman 1998] J. Erlijman, "New subfactors from braid group representations", Trans. Amer. Math. Soc. 350:1 (1998), 185-211. MR 98k:46100 Zbl 0887.46035

[Erlijman and Wenzl 2007] J. Erlijman and H. Wenzl, "Subfactors from braided $C^{*}$ tensor categories", Pacific J. Math. 231:2 (2007), 361-399. MR 2008g:46111 Zbl 1152.46051

[Evans and Gannon 2010] D. E. Evans and T. Gannon, "Modular invariants and twisted equivariant $K$-theory II: Dynkin diagram symmetries”, preprint, 2010. arXiv 1012.1634

[Evans and Kawahigashi 1994] D. E. Evans and Y. Kawahigashi, "Orbifold subfactors from Hecke algebras”, Comm. Math. Phys. 165:3 (1994), 445-484. MR 96c:46059 Zbl 0805.46077

[Evans and Kawahigashi 1998] D. E. Evans and Y. Kawahigashi, Quantum symmetries on operator algebras, Oxford Mathematical Monographs, The Clarendon Press, Oxford University Press, New York, 1998. MR 99m:46148 Zbl 0924.46054

[Evans and Pugh 2011] D. E. Evans and M. Pugh, "Pugh, Mathew, Braided subfactors, spectral measures, planar algebras and Calabi-Yau algebras associated to SU(3) modular invariants", preprint, 2011. arXiv 1110.4547

[Fuchs and Schweigert 2000] J. Fuchs and C. Schweigert, "Solitonic sectors, $\alpha$-induction and symmetry breaking boundaries", Phys. Lett. B 490 (2000), 163-172. MR 2001k:81222 Zbl 1031.81605

[Gaberdiel and Gannon 2002] M. R. Gaberdiel and T. Gannon, "Boundary states for WZW models", Nuclear Phys. B 639:3 (2002), 471-501. MR 2003j:81192 Zbl 0997.81052

[Goodman and Wenzl 1990] F. M. Goodman and H. Wenzl, "Littlewood-Richardson coefficients for Hecke algebras at roots of unity”, Adv. Math. 82:2 (1990), 244-265. MR 91i:20013 Zbl 0714.20004

[Goodman et al. 1989] F. M. Goodman, P. de la Harpe, and V. F. R. Jones, Coxeter graphs and towers of algebras, Mathematical Sciences Research Institute Publications 14, Springer, New York, 1989. MR 91c:46082 Zbl 0698.46050

[Iorgov and Klimyk 2005] N. Z. Iorgov and A. U. Klimyk, "Classification theorem on irreducible representations of the $q$-deformed algebra $U_{q}^{\prime}\left(\mathrm{so}_{n}\right)$ ", Int. J. Math. Math. Sci. 2 (2005), 225-262. MR 2006c:17021 Zbl 1127.17016

[Jones 1983] V. F. R. Jones, "Index for subfactors", Invent. Math. 72:1 (1983), 1-25. MR 84d:46097 Zbl 0508.46040

[Kac 1990] V. G. Kac, Infinite-dimensional Lie algebras, 3rd ed., Cambridge University Press, 1990. MR 92k:17038 Zbl 0716.17022

[Koike 1997] K. Koike, "Principal specializations of the classical groups and $q$-analogs of the dimension formulas”, Adv. Math. 125:2 (1997), 236-274. MR 98f:20027 Zbl 0867.22013

[Koike and Terada 1987] K. Koike and I. Terada, "Young-diagrammatic methods for the representation theory of the classical groups of type $B_{n}, C_{n}, D_{n}$ ", J. Algebra 107:2 (1987), 466-511. MR 88i:22035 Zbl 0622.20033

[Letzter 1997] G. Letzter, "Subalgebras which appear in quantum Iwasawa decompositions", Canad. J. Math. 49:6 (1997), 1206-1223. MR 99g:17022 Zbl 0898.17005

[Letzter 2002] G. Letzter, "Coideal subalgebras and quantum symmetric pairs", pp. 117-165 in New directions in Hopf algebras, edited by S. Montgomery and H.-J. Schneider, Math. Sci. Res. Inst. Publ. 43, Cambridge Univ. Press, 2002. MR 2003g:17025 Zbl 1025.17005

[Molev 2003] A. I. Molev, “A new quantum analog of the Brauer algebra: Quantum groups and integrable systems”, Czechoslovak J. Phys. 53:11 (2003), 1073-1078. MR 2005e:17024 
[Noumi 1996] M. Noumi, "Macdonald's symmetric polynomials as zonal spherical functions on some quantum homogeneous spaces", Adv. Math. 123:1 (1996), 16-77. MR 98a:33004 Zbl 0874.33011

[Ocneanu 2002] A. Ocneanu, "The classification of subgroups of quantum SU $(N)$ ", pp. 133-159 in Quantum symmetries in theoretical physics and mathematics (Bariloche, 2000), edited by R. Coquereaux et al., Contemp. Math. 294, Amer. Math. Soc., Providence, RI, 2002. MR 2003h:81101 Zbl 1193.81055

[Petkova and Zuber 2002] V. Petkova and J.-B. Zuber, "Conformal field theories, graphs and quantum algebras", pp. 415-435 in MathPhys odyssey, 2001, edited by M. Kashiwara and T. Miwa, Prog. Math. Phys. 23, Birkhäuser, Boston, MA, 2002. MR 1903984 Zbl 1026.81053

[Ram and Wenzl 1992] A. Ram and H. Wenzl, "Matrix units for centralizer algebras", J. Algebra 145:2 (1992), 378-395. MR 93g:16024 Zbl 0761.16008

[Sunder 1987] V. S. Sunder, "A model for AF algebras and a representation of the Jones projections", J. Operator Theory 18:2 (1987), 289-301. MR 89e:46079 Zbl 0693.46054

[Verrill 2001] R. Verrill, Positive energy representations of $L^{\sigma} S U(2 r)$ and orbifold fusion, thesis, University of Cambridge, 2001.

[Wassermann 2010] A. Wassermann, "Subfactors and Connes fusion for twisted loop groups", preprint, 2010. arXiv 1003.2292

[Wenzl 1988a] H. Wenzl, "Hecke algebras of type $A_{n}$ and subfactors", Invent. Math. 92:2 (1988), 349-383. MR 90b:46118 Zbl 0663.46055

[Wenzl 1988b] H. Wenzl, "On the structure of Brauer's centralizer algebras", Ann. of Math. (2) 128:1 (1988), 173-193. MR 89h:20059 Zbl 0656.20040

[Wenzl 2011] H. Wenzl, "Quotients of representation rings”, Represent. Theory 15 (2011), 385-406. MR 2012i:22019 Zbl 06043183

[Wenzl 2012] H. Wenzl, “A q-Brauer algebra”, J. Algebra 358 (2012), 102-127. MR 2905021

[Weyl 1997] H. Weyl, The classical groups: Their invariants and representations, Princeton Landmarks in Mathematics, Princeton University Press, 1997. MR 98k:01049 Zbl 1024.20501

[Xu 1998] F. Xu, "New braided endomorphisms from conformal inclusions", Comm. Math. Phys. 192:2 (1998), 349-403. MR 99b:81139 Zbl 0908.46044

[Xu 2009] F. Xu, "On affine orbifold nets associated with outer automorphisms", Comm. Math. Phys. 291:3 (2009), 845-861. MR 2011d:46141 Zbl 1186.81108

Received July 19, 2011.

HANS WENZL

DEPARTMENT OF MATHEMATICS

UNIVERSITY OF CALIFORNIA, SAN DiEgo

9500 GILMAN DRIVE

DEPT 0112

LA JOLLA CA 92093-0112

UNITED STATES

hwenzl@ucsd.edu 


\title{
PACIFIC JOURNAL OF MATHEMATICS
}

\author{
http://pacificmath.org \\ Founded in 1951 by \\ E. F. Beckenbach (1906-1982) and F. Wolf (1904-1989)
}

\section{EDITORS}

V. S. Varadarajan (Managing Editor)

Department of Mathematics

University of California

Los Angeles, CA 90095-1555

pacific@math.ucla.edu

Vyjayanthi Chari

Department of Mathematics

University of California

Riverside, CA 92521-0135

chari@math.ucr.edu

\section{Robert Finn}

Department of Mathematics Stanford University

Stanford, CA 94305-2125

finn@math.stanford.edu

Kefeng Liu

Department of Mathematics

University of California

Los Angeles, CA 90095-1555

liu@math.ucla.edu
Darren Long

Department of Mathematics

University of California

Santa Barbara, CA 93106-3080

long@math.ucsb.edu

Jiang-Hua Lu

Department of Mathematics

The University of Hong Kong

Pokfulam Rd., Hong Kong jhlu@maths.hku.hk

Alexander Merkurjev

Department of Mathematics

University of California

Los Angeles, CA 90095-1555

merkurev@math.ucla.edu
Sorin Popa

Department of Mathematics University of California

Los Angeles, CA 90095-1555 popa@math.ucla.edu

Jie Qing

Department of Mathematics

University of California

Santa Cruz, CA 95064

qing@cats.ucsc.edu

Jonathan Rogawski

Department of Mathematics

University of California

Los Angeles, CA 90095-1555

jonr@math.ucla.edu

\section{PRODUCTION}

pacific@math.berkeley.edu

\section{SUPPORTING INSTITUTIONS}

ACADEMIA SINICA, TAIPEI

CALIFORNIA INST. OF TECHNOLOGY INST. DE MATEMÁTICA PURA E APLICADA KEIO UNIVERSITY

MATH. SCIENCES RESEARCH INSTITUTE NEW MEXICO STATE UNIV.

OREGON STATE UNIV.

\author{
STANFORD UNIVERSITY \\ UNIV. OF BRITISH COLUMBIA \\ UNIV. OF CALIFORNIA, BERKELEY \\ UNIV. OF CALIFORNIA, DAVIS \\ UNIV. OF CALIFORNIA, LOS ANGELES \\ UNIV. OF CALIFORNIA, RIVERSIDE \\ UNIV. OF CALIFORNIA, SAN DIEGO \\ UNIV. OF CALIF., SANTA BARBARA
}

\author{
UNIV. OF CALIF., SANTA CRUZ \\ UNIV. OF MONTANA \\ UNIV. OF OREGON \\ UNIV. OF SOUTHERN CALIFORNIA \\ UNIV. OF UTAH \\ UNIV. OF WASHINGTON \\ WASHINGTON STATE UNIVERSITY
}

These supporting institutions contribute to the cost of publication of this Journal, but they are not owners or publishers and have no responsibility for its contents or policies.

See inside back cover or pacificmath.org for submission instructions.

The subscription price for 2012 is US \$420/year for the electronic version, and \$485/year for print and electronic.

Subscriptions, requests for back issues from the last three years and changes of subscribers address should be sent to Pacific Journal of Mathematics, P.O. Box 4163, Berkeley, CA 94704-0163, U.S.A. Prior back issues are obtainable from Periodicals Service Company, 11 Main Street, Germantown, NY 12526-5635. The Pacific Journal of Mathematics is indexed by Mathematical Reviews, Zentralblatt MATH, PASCAL CNRS Index, Referativnyi Zhurnal, Current Mathematical Publications and the Science Citation Index.

The Pacific Journal of Mathematics (ISSN 0030-8730) at the University of California, c/o Department of Mathematics, 969 Evans Hall, Berkeley, CA 94720-3840, is published monthly except July and August. Periodical rate postage paid at Berkeley, CA 94704, and additional mailing offices. POSTMASTER: send address changes to Pacific Journal of Mathematics, P.O. Box 4163, Berkeley, CA 94704-0163.

PJM peer review and production are managed by EditFLOW ${ }^{\mathrm{TM}}$ from Mathematical Sciences Publishers.

PUBLISHED BY PACIFIC JOURNAL OF MATHEMATICS

at the University of California, Berkeley 94720-3840

A NON-PROFIT CORPORATION

Typeset in LATEX

Copyright $(02012$ by Pacific Journal of Mathematics 


\section{PACIFIC JOURNAL OF MATHEMATICS}

Volume $259 \quad$ No. $2 \quad$ October 2012

Flag subdivisions and $\gamma$-vectors

257

Christos A. ATHANASIAdis

Rays and souls in von Mangoldt planes

279

IGOR BELEGRADEK, ERIC CHOI and NOBUHIRO INNAMI

Isoperimetric surfaces with boundary, II

ABRAHAM FRANDSEN, DONALD SAMPSON and NEIL

STEINBURG

Cyclic branched coverings of knots and quandle homology

YUICHI KABAYA

On a class of semihereditary crossed-product orders

JOHN S. KAUTA

An explicit formula for spherical curves with constant torsion

DEMETRE KAZARAS and IVAN STERLING

Comparing seminorms on homology

JEAN-FranÇOIS LAFONT and CHRISTOPHE PITTET

Relatively maximum volume rigidity in Alexandrov geometry

NAN LI and XIAOCHUN RONG

Properness, Cauchy indivisibility and the Weil completion of a group of 421 isometries

Antonios Manoussos and Polychronis Strantzalos

Theta lifts of strongly positive discrete series: the case of $(\tilde{\mathrm{Sp}}(n), O(V)) 445$

IVAN MATIĆ

Tunnel one, fibered links

MATt RATHBUN

Fusion symmetric spaces and subfactors

HANS WENZL 